\title{
Frequency planning for clustered jointly processed Cellular Multiple Access Channel
}

\begin{tabular}{|c|c|}
\hline Journal: & IET Communications \\
\hline Manuscript ID: & Draft \\
\hline Manuscript Type: & Research Paper \\
\hline Date Submitted by the Author: & $n / a$ \\
\hline Complete List of Authors: & $\begin{array}{l}\text { Majid, Muhammad Imran; Centre for Communication Systems Research; } \\
\text { Imran, Muhammad; University of Surrey, CCSR } \\
\text { Hoshyar, Reza; University of Surrey, Electronics Engg }\end{array}$ \\
\hline Keyword: & $\begin{array}{l}\text { INFORMATION THEORY, WIRELESS ACCESS NETWORKS (WANS), GENETIC } \\
\text { ALGORITHMS, FREQUENCY ALLOCATION }\end{array}$ \\
\hline \multicolumn{2}{|c|}{$\begin{array}{l}\text { Note: The following files were submitted by the author for peer review, but cannot be converted to } \\
\text { PDF. You must view these files (e.g. movies) online. }\end{array}$} \\
\hline $\begin{array}{l}\text { refer_phd2.bib } \\
\text { jrnl_contributions3.tex }\end{array}$ & \\
\hline
\end{tabular}




\title{
Frequency planning for clustered jointly processed Cellular Multiple Access Channel
}

\author{
Muhammad I. Majid, Muhammad A. Imran, Reza Hoshyar \\ Centre for Communications and Systems Research \\ University of Surrey, Guildford, \\ GU2 7XH, United Kingdom \\ Email:imran.majid@gmail.com,m.imran, r.hoshyar@surrey.ac.uk
}

\begin{abstract}
Due to limited resources, it is hard to guarantee minimum service levels to all users in conventional cellular systems. Although global cooperation of Access Points (APs) is considered promising, practical means of enhancing efficiency of cellular systems is by considering distributed or clustered jointly processed APs. Authors present a novel 'QoS balancing scheme' to maximize sum rate as well as achieve cell based fairness for clustered jointly processed cellular Multiple Access Channel (CC-CMAC). Closed form cell level quality of service (QoS) balancing function is derived. Maximization of this function is proved as an NP Hard problem. Hence, using power-frequency granularity, a modified genetic algorithm (GA) is proposed. For $I S D<500 \mathrm{~m}$, results show that with no fairness considered, the upper bound of the capacity region is achievable. Applying hard fairness restraints on users transmitting in moderately dense AP system, $20 \%$ reduction in sum rate contribution increases fairness by upto $10 \%$. The flexible QoS can be applied onto a GA based centralized dynamic frequency planner architecture.
\end{abstract}

\section{Introduction}

Global AP cooperation which has been studied in classical Wyner model [1], extended with fading [2] and distance dependent pathloss [3], is considered too complex to implement. Recently, localized joint processing of APs has been proposed in the framework of isolated groups [4], local message passing [5, 6], and limited backhaul [7]. In [8], the concept of rate splitting from Interference Channel were applied to clustered jointly processed APs. In such schemes, Inter Cluster Interference from users outside cluster is the only dominant type of interference. Since edge cells are more prone to interference from neighbouring cells, the level of interference at the edge and centre of a cluster is not the same [9]. Hence techniques involving strongest channel coefficients like dynamic clustering [10] are proposed to address the varying degree of interference levels. In this work however we consider geographically fixed clusters.

Similar to conventional cellular networks, frequency planning can be considered an effective method to control the interference situation. Here we refer to such systems as cluster cooperative cellular multiple access channels or (CC-CMAC) [11]. We consider the frequency planning for CC-CMAC in order to maximize network utility using a derived formulation of per cell sum rate. We consider balancing of per cell sum rate within cell users in a 7 cell cluster surrounded by 6 first tier interfering clusters and so on. In this context per cell sum rate balancing takes place at the cluster head. Cumulatively, the network wide balancing of per cell sum rate for users transmitting in a CC-CMAC is referred to as QoS balancing function. This balancing approach is useful in quantifying cell utility for a range of per cell 
sum rate conditions. Two cases of interest are : (a) maximizing sum rate, and (b) achieving cell based fairness.

We take a cell based approach as in [12] and assume a medium-term time scale corresponding to cell-level load variations. The short term variations related to user mobility and instantaneous channel conditions are assumed to be handled by each cluster's radio resource management functionality.

The rest of the paper is organized as follows. Section 2 introduces the concept of CC-CMAC and bin allocation over AP based fixed clusters and presents the system model. The system architecture is presented in Section sec:problemformulation. In Section 4, the closed form representation of cell based QoS balancing function for CC-CMAC is derived. In Section 5, the solution to this formulation is proved to be NP Hard. Section 6 discusses the solution framework using a modified heuristic i.e. genetic algorithm(GA). The effect of power-frequency granularity, rate and fairness are discussed in Section 7. The application of CC-CMAC in a practical GA based architecture, optimizing QoS balancing function, with architecture complexity analysisis presented in Section 7.2. We conclude in Section 8.

\section{Notation}

Lower and upper case boldface symbols denote vectors and matrices, respectively; Math Curl represents the set notation, $(.)^{\dagger}$ denotes the Hermitian transpose, |.| represents the cardinality of a set, diag is the diagonal of the matrix, $t r$ represents the matrix trace and $\mathbb{E}[$.$] represents the expectation operator.$

\section{System Model}

The uplink capacity of CC-CMAC is analyzed using bin allocation. Bins are disjoint equal width frequency bands with flat transmit power spectral density used over them [12]. Specifically, a hexagonal grid of $N$ cells is assumed which is divided into $Q$ fixed, equal and identical clusters. $\mathcal{N}_{q}$ is the set of all cells belonging to cluster $q$, where $q=1, \ldots, Q, \&\left|\mathcal{N}_{q}\right|=\frac{N}{Q}$. Similarly, $\overline{\mathcal{N}_{q}}$ is the set of all cells not belonging to cluster, $q$ where $\left|\overline{\mathcal{N}_{q}}\right|=N-\frac{N}{Q}$. Noise is AWGN. For transmission over a given bin $b$, the $\left|\mathcal{N}_{q}\right| \times 1$ received signal vector $\mathbf{y}^{q}$ for the $q^{\text {th }}$ cluster can be modelled as:

$$
\begin{aligned}
& \mathbf{y}^{q}=\mathbf{H}^{q} \mathbf{x}^{q}+\hat{\mathbf{z}}^{q} \\
& \hat{\mathbf{z}}^{q}=\hat{\mathbf{H}}^{q} \hat{\mathbf{x}}^{q}+\mathbf{z}^{q}
\end{aligned}
$$

where the $\left|\mathcal{N}_{q}\right| \times K\left|\mathcal{N}_{q}\right|$ channel matrix, $\mathbf{H}^{q}=\left[\mathbf{H}_{1}^{q}, \ldots, \mathbf{H}_{\left|\mathcal{N}_{q}\right|}^{q}\right]$, contains complex gain matrices for $K$ users per cell, within the $\left|\mathcal{N}_{q}\right|$ cells which are located within $q^{\text {th }}$ cluster and $\left|\mathcal{N}_{q}\right| \times K\left(N-\left|\mathcal{N}_{q}\right|\right)$ channel matrix, $\hat{\mathbf{H}}^{q}=\left[\hat{\mathbf{H}}_{1}^{q}, \ldots, \hat{\mathbf{H}}_{N-\left|\mathcal{N}_{q}\right|}^{q}\right]$, contains complex gain matrices for all $N-\left|\mathcal{N}_{q}\right|$ cells out of the $q^{\text {th }}$ cluster. Similarly, $\mathbf{x}^{q}=\left[\mathbf{x}_{1}^{q}, \ldots, \mathbf{x}_{\left|\mathcal{N}_{q}\right|}^{q}\right]^{T}$ is a $K\left|\mathcal{N}_{q}\right| \times 1$ transmit symbol vector for all cells within $q^{t h}$ cluster and $\hat{\mathbf{x}}^{q}=\left[\hat{\mathbf{x}}_{1}^{q}, \ldots, \hat{\mathbf{x}}_{N-\left|\mathcal{N}_{q}\right|}^{q}\right]^{T}$ is a $K\left(N-\left|\mathcal{N}_{q}\right|\right) \times 1$ transmit symbol vector for all cells outside cluster $q . \mathbf{z}^{q}$ represents the $\left|\mathcal{N}_{q}\right| \times 1$ independent complex circularly symmetric AWGN vector at receiver. The transmitters are subject to power constraint $\operatorname{tr}\left(\mathbb{E}\left[\mathbf{x}_{i}^{q}\left(\mathbf{x}_{i}^{q}\right)^{\dagger}\right]\right) \leq K P_{i} \& \operatorname{tr}\left(\mathbb{E}\left[\hat{\mathbf{x}}_{j}^{q}\left(\hat{\mathbf{x}}_{j}^{q}\right)^{\dagger}\right]\right) \leq K P_{j}$, where cell $i$ and cell $j$ are located within and outside cluster, $q$ respectively. We assume that the users have same per user power constraint. Hence $P_{i}=P_{j}=P_{n}, \forall n=1, \ldots, N$. This has applications in energy constrained transmitter, where power is restricted over the entire spectrum.

A simple scenario for 2 cells transmitting to APs in 2 different clusters is explained 
in Figure 1. We consider allocating sets of frequency bins to each cell in a cluster as per the bin allocation shown in the bottom of Figure 1.

Let $a_{n, b}$ be the $n^{\text {th }}$ row and $b^{\text {th }}$ column entry for the allocation matrix, A. Allocation matrix $\mathbf{A}$ represents allocation of $b^{\text {th }}$ bin to users in $n^{\text {th }}$ cell (Non zero integer implies allocation; 0 implies otherwise). If allocation matrix for users transmitting in Cell 1 in Figure 1 is produced it will have values of $P(1,1)$ at $a_{1,1}$ and so on. The maximum bins (discrete frequency allocation intervals) that are implemented are $B$ bins. For $V$ quantization bits per bin, $\mathbf{A} \in\left\{1, \ldots, 2^{V}-1\right\}^{N \times B}$ represents joint bin and power allocation to the $N$ cells in the system. Here $\mathcal{N}_{b}(\mathbf{A})$ represents the set of cells which have been allocated the $b^{t h}$ bin.

\section{System Architecture}

As shown in Figure 2, GA based network QoS balancing function (discussed in Section 3.1) is implemented in a centralized architecture. The cell path loss information is in the form of $\overline{\varsigma_{1}^{q}}$ for the transmission from cell indexed by 1 and whose signal is received at the AP in the $q^{\text {th }}$ cell. This information can be represented with minimum algorithmic complexity with the help of closed form framework of bin based allocation in CC-CMAC. This is explored in Section 4. The filtering function is represented by $g(\cdot)$ which takes $\hat{\mathbf{A}}$ and $\overline{\boldsymbol{\zeta}_{n}^{q}}$ as inputs. It assigns the updated frequency allocation algorithm $\hat{\mathbf{A}}$ to the network. Further, the updated $\overline{\boldsymbol{\zeta}_{n}^{q}}$ is sent to Central Frequency Planner (CFP). Here for the first run, $\hat{\mathbf{A}}$ consists of randomly generated bin allocation matrix, A. Subsequently, the GA entity assigns the fittest solution to $\hat{\mathbf{A}}$. These entries are the result of partial bin reuse (PBR) solution framework which are discussed in Section 6 . In this context, $\overline{\boldsymbol{\zeta}_{n}^{q}}$ is collected every hour so as to represent the changing dynamics of user profiles. This has applications in Collingwood Circle design, and random non homogeneous user traffic profile as detailed in [11].

\subsection{Network wide QoS balancing Framework}

Mobile network operators would like to ascertain network wide efficiency criterion to meet objectives of profit maximization. Hence the need for a system wide efficiency criterion.In this section we determine this efficiency in terms of QoS balancing of per cell sum rate. For a given fairness coefficient $\gamma, U_{\gamma}(\mathbf{A})$ is the system level QoS balance metric which is broken down to cell based QoS balance metric in (3). Mathematically,

$$
\begin{gathered}
U_{\gamma}(\mathbf{A})=\frac{1}{N} \sum_{q=1}^{Q} \sum_{n \in \mathcal{N}_{q}} u_{\gamma}\left(R_{n, q}(\mathbf{A})\right) \\
\forall b=1, \ldots, B ; \quad \forall n=1, \ldots, N
\end{gathered}
$$

Here $u_{\gamma}\left(R_{n, q}(\mathbf{A})\right)$ is the QoS balancing metric for users in $n^{\text {th }}$ cell within the $q^{\text {th }}$ cluster. Here for a given $\mathbf{A}, R_{n, q}(\mathbf{A})$ is the sum rate due to transmission from the $n^{\text {th }}$ cell within the $q^{\text {th }}$ cluster. Our objective is to,

$$
\begin{array}{r}
\max U_{\gamma}(\mathbf{A}) \\
\text { s.t. } \quad R_{n, q}(\mathbf{A}) \in \mathbb{R}^{+}
\end{array}
$$


subject to the power constraints as embodied in A. Combining (3) and (4), the optimization problem is to maximize network wide QoS balancing function. Hence,

$$
\begin{gathered}
\overbrace{\mathbf{A}} \overbrace{N \sum_{q=1}^{Q}(\overbrace{\sum_{n_{\in} \mathcal{N}_{q}}\left[u_{\gamma}\left(R_{n, q}(\mathbf{A})\right)\right]}^{\text {Cell based QoS balancing } \text { q }^{\text {th }} \text { cluster }})}^{\text {System wide cell based QoS balancing }}) \\
\text { s.t. } R_{n, q}(\mathbf{A}) \in \mathbb{R}^{+} \\
\quad \sum_{b=1}^{B} P_{n, b} \leq P \\
\forall n=1, \ldots, N ; \forall b=1, \ldots, B .
\end{gathered}
$$

where $P$ is the maximum per user transmit power over all the allocated bins. The value of $P_{n, b}$ varies across bins and across different cell users within a cluster. This is because QoS balancing needs to account for two forms of interference i.e. inter cluster and intra cluster interferences. This has implications for considering such a formulation for macro cells in conjunction with pico cells and femto cells. However, since our fomulation considers fixed cluster and is not a hierarchical based clustering, further topics likes HetNet can be modelled but not explored in detail for in this paper. The bin based CC-CMAC formulation has been discussed in [13] and performance detailed in [11]. In this paper, the authors build on the material from their previous work.

\section{Cell based QoS Balancing Function}

$U_{\gamma}$ depends on $u_{\gamma}$, which is a function of cell based sum rate, $R_{n, q}(\mathbf{A})$. We first derive a closed form representation of per cluster sum rate from the iterative simulation based formula. The closed form expression has the advantage of reduced complexity and signalling cost as compared to the averaged simulation based sum rate expression. The jointly processed sum rate for $q^{\text {th }}$ cluster is then decoded to analyze the sum rate contribution by users in every cell located within $q^{\text {th }}$ cluster. This is implemented using MMSE SIC framework [14].

\subsection{Simulation based sum rate for CC-CMAC}

The concept of joint processing of signals to evaluate sum rate was introduced in Wyner's Gaussian Cellular Multiple Access Channel (GCMAC) model [1]. Letzepis extended classical work of Wyner, to produce a log det formulation for capacity of jointly processed cellular networks with free space path loss. The sum rate representation for $q^{\text {th }}$ geographical fixed cluster of APs as in [13] was denoted using the expectation of per cell sum rate formulation : $R_{q}=\log \operatorname{det}\left(\frac{\mathbf{S}_{\mathbf{q}}}{\mathbf{S}_{\mathbf{n q}}}\right)$ The useful signal is represented in numerator $\left(\mathbf{S}_{\mathbf{q}}\right)$ and interference as well as noise in demoninator $\left(\mathbf{S}_{\mathbf{n q}}\right)$. Extending the log det formulation, in this section this formulation is explained and extended to represent a closed form representation of per cell sum rate to accomodate into our proposed architecture (Figure 2).

As per simulation results of $\mathbb{E}\left[R_{q}(\mathbf{A})\right]$ versus $P_{n}(\mathbf{A}) ; \forall n, R_{q}(\mathbf{A})$ is a concave 
function [11]. Hence, applying the Jensen's inequality [15] gives the following:

$$
\mathbb{E}\left[R_{q}(\mathbf{A})\right] \leq \frac{1}{B} \sum_{b=1}^{B} \log \left[\mathbb{E}\left(\frac{\operatorname{det}\left(\sigma_{z}^{2} \mathbf{I}_{\frac{N}{Q}}+\sum_{n \in \mathcal{N}_{b}(\mathbf{A})} P_{n}(\mathbf{A}) \mathbf{H}_{n}^{q}\left(\mathbf{H}_{n}^{q}\right)^{\dagger}\right)}{\operatorname{det}\left(\sigma_{z}^{2} \mathbf{I}_{\frac{N}{Q}}+\sum_{n \in \mathcal{N}_{b}(\mathbf{A}) \cap \overline{\mathcal{N}_{q}}} P_{n}(\mathbf{A}) \mathbf{H}_{n}^{q}\left(\mathbf{H}_{n}^{q}\right)^{\dagger}\right)}\right]\right]
$$

In (7), assume that $K \rightarrow \infty$. As per law of large numbers, $\mathbb{E}\left[\frac{X}{Y}\right]=\frac{\mathbb{E}[X]}{\mathbb{E}[Y]}$, since large $K$ implies a deterministic $X$ and $Y$. Further, using $\log \left(A B^{-1}\right)=\log (A)-$ $\log (B)$ the following can be deduced:

$$
\begin{aligned}
\mathbb{E}\left[R_{q}(\mathbf{A})\right] \leq & \frac{1}{B} \sum_{b=1}^{B}\left[\log \left\{\mathbb{E}\left[\operatorname{det}\left(\sigma_{z}^{2} \mathbf{I}_{\frac{N}{Q}}+\sum_{n \in \mathcal{N}_{b}(\mathbf{A})} P_{n}(\mathbf{A}) \mathbf{H}_{n}^{q}\left(\mathbf{H}_{n}^{q}\right)^{\dagger}\right)\right]\right\}\right. \\
& \left.-\log \left\{\mathbb{E}\left[\operatorname{det}\left(\sigma_{z}^{2} \mathbf{I}_{\frac{N}{Q}}+\sum_{n \in \mathcal{N}_{b}(\mathbf{A}) \cap \overline{\mathcal{N}_{q}}} P_{n}(\mathbf{A}) \mathbf{H}_{n}^{q}\left(\mathbf{H}_{n}^{q}\right)^{\dagger}\right)\right]\right\}\right]
\end{aligned}
$$

Consider a fast fading scenario where each fading coefficient is represented by $g_{n, k}^{n_{q}}$ and pathloss identified by $s_{n, k}^{n_{q}}$, for transmission from $k^{t h}$ user in $n^{t h}$ cell to AP in cell $n_{q}$ located within cluster $q$. The channel coefficient is represented by $h_{n, k}^{n_{q}}=g_{n, k}^{n_{q}} \odot s_{n, k}^{n_{q}}$ where $\odot$ represents the Hadamard product. The transmission is modelled as a time-varying ergodic process.

Assuming a large number of users per cell i.e. $K \rightarrow \infty$, as per law of large numbers, $1 / K \sum_{k=1}^{K}\left|g_{n, k}^{n_{q}}\right|^{2} \rightarrow 1$ for $\forall n, \forall q$. Using complex matrices for fading, product of complex fading coefficients with its complex conjugate is equal to power which is normalized to unity. Hence,

$$
\mathbb{E}\left[g_{n, k}^{n_{q}} g_{n, k}^{n_{q} \dagger}\right]=\mathbb{E}\left[\left(g_{n, k}^{n_{q}}\right)^{2}\right]=1
$$

Moreover, the expectation of product of a complex fading coefficient with the complex conjugate of a different fading coefficient but following the same PDF is the square of the expected value of an individual fading coefficient. Hence,

$$
\mathbb{E}\left[g_{n, k}^{n_{q}} g_{n^{\prime}, k^{\prime}}^{n_{q}{ }^{\dagger}}\right]=\left[\mu_{g}\right]^{2}
$$

here $k^{\prime} \neq k$ and $n^{\prime} \neq n$. $\mu_{g}$ is the expected value of an individual fading coefficient. In the case of the Rayleigh based flat fading, $\mu_{g}=0$ as in [2].

Further, define $\mathbf{g}_{n}^{n_{q}}=\left[g_{n, 1}^{n_{q}}, g_{n, 2}^{n_{q}}, \ldots, g_{n, K}^{n_{q}}\right]$ and $\mathbf{s}_{n}^{n_{q}}=\left[s_{n, 1}^{n_{q}}, s_{n, 2}^{n_{q}}, \ldots, s_{n, K}^{n_{q}}\right]$ as $1 \times K$ complex fading vector and $1 \times K$ deterministic pathloss vector for transmission from users in $n^{\text {th }}$ cell and received at the AP in the $n_{q}^{\text {th }}$ cell within the $q^{\text {th }}$ cluster.

Considering only the diagonal entries of the estimation for covariance of $\mathbf{H}_{n}^{q}$, the following can be deduced:

$$
\begin{aligned}
\operatorname{diag}\left(\mathbb{E}\left[\mathbf{H}_{n}^{q} \mathbf{H}_{n}^{q \dagger}\right]\right) & =\mathbb{E}\left[\left(\mathbf{s}_{n}^{1} \odot \mathbf{g}_{n}^{1}\right)\left(\mathbf{s}_{n}^{1} \odot \mathbf{g}_{n}^{1}\right)^{\dagger}, \ldots,\left(\mathbf{s}_{n}^{\left|\mathcal{N}_{q}\right|} \odot \mathbf{g}_{n}^{\left|\mathcal{N}_{q}\right|}\right)\left(\mathbf{s}_{n}^{\left|\mathcal{N}_{q}\right|} \odot \mathbf{g}_{n}^{\left|\mathcal{N}_{q}\right| \gamma \dagger} \mid 1\right)\right. \\
& =\mathbb{E}\left[\left(\mathbf{s}_{n}^{1} \mathbf{s}_{n}^{1 \dagger}\right) \odot\left(\mathbf{g}_{n}^{1} \mathbf{g}_{n}^{1 \dagger}\right), \ldots,\left(\mathbf{s}_{n}^{\left|\mathcal{N}_{q}\right|} \mathbf{s}_{n}^{\left|\mathcal{N}_{q}\right|^{\dagger}}\right) \odot\left(\mathbf{g}_{n}^{\left|\mathcal{N}_{q}\right|} \mathbf{g}_{n}^{\left|\mathcal{N}_{q}\right|^{\dagger}}\right)\right] \\
& =\left[\mathbb{E}\left[\mathbf{s}_{n}^{1} \mathbf{s}_{n}^{1 \dagger}\right], \ldots, \mathbb{E}\left[\mathbf{s}_{n}^{\left|\mathcal{N}_{q}\right|} \mathbf{s}_{n}^{\left|\mathcal{N}_{q}\right| \dagger}\right]\right] \\
& =\left[\overline{\varsigma_{n}^{1}}, \ldots, \overline{\varsigma_{n}^{\left|\mathcal{N}_{q}\right|}}\right]
\end{aligned}
$$


Here $\operatorname{diag}($.$) is the diagonal entry of the matrix in the argument. (13) is derived$ from (12) after combining with (9). Further, $\overline{\boldsymbol{\varsigma}_{n}^{q}}=\left[\overline{\varsigma_{n}^{1}}, \ldots, \overline{\varsigma_{n}^{\left|\mathcal{N}_{q}\right|}}\right]$ is the $1 \times\left|\mathcal{N}_{q}\right|$ deterministic vector representing average pathloss coefficient, $\overline{\varsigma_{n}^{n_{q}}}$ experienced by users in $n^{t h}$ cell transmitting to all APs in $q^{t h}$ cluster. Hence, $\overline{\varsigma_{n}^{n_{q}}} \triangleq \frac{1}{K} \sum_{k=1}^{K}\left(s_{n, k}^{n_{q}}\right)^{2}$ $\forall n_{q}=1, \ldots,\left|\mathcal{N}_{q}\right|$. Extending the above to the formulation for power and bin allocation, the following can be deduced for asymptotically large number of users:

$$
\operatorname{diag}\left(\mathbb{E}\left[P_{n}(\mathbf{A}) \mathbf{H}_{n}^{q}\left(\mathbf{H}_{n}^{q}\right)^{\dagger}\right]\right)=P_{n}(\mathbf{A}) \overline{\boldsymbol{\varsigma}_{n}^{q}}
$$

Recalling (8) and decomposing the RHS log argument, it is known that

$$
\begin{array}{r}
W_{1}=\mathbb{E}\left[\operatorname{det}\left(\sigma_{z}^{2} \mathbf{I}_{\frac{N}{Q}}+\sum_{n \in \mathcal{N}_{b}(\mathbf{A})} P_{n}(\mathbf{A}) \mathbf{H}_{n}^{q}\left(\mathbf{H}_{n}^{q}\right)^{\dagger}\right)\right] \\
W_{2}=\mathbb{E}\left[\operatorname{det}\left(\sigma_{z}^{2} \mathbf{I}_{\frac{N}{Q}}+\sum_{n \in \mathcal{N}_{b}(\mathbf{A}) \cap \overline{\mathcal{N}_{q}}} P_{n}(\mathbf{A}) \mathbf{H}_{n}^{q}\left(\mathbf{H}_{n}^{q}\right)^{\dagger}\right)\right]
\end{array}
$$

Plugging (15) in (17),

$$
W_{1}=\operatorname{det}\left[\begin{array}{ccc}
\sigma_{z}^{2}+\sum_{n \in \mathcal{N}_{b}(\mathbf{A})} P_{n}(\mathbf{A}) \overline{\varsigma_{n}^{1}} & 0 & 0 \\
\vdots & \ddots & \vdots \\
0 & 0 & \sigma_{z}^{2}+\sum_{n \in \mathcal{N}_{b}(\mathbf{A})} P_{n}(\mathbf{A}) \overline{\varsigma_{n}^{\left|\mathcal{N}_{q}\right|}}
\end{array}\right]
$$

Applying similar concept to argument of second log on RHS of (8), and summing up for all $Q$ clusters, the closed form sum rate formulation for CC-CMAC, is as follows:

$$
\begin{aligned}
\bar{R}(\mathbf{A}) \leq & \sum_{q=1}^{Q}\left\{\frac { 1 } { B } \sum _ { b = 1 } ^ { B } \left[\log \left(\prod_{n_{q} \in \mathcal{N}_{q}}\left[\sigma_{z}^{2}+\sum_{n \in \mathcal{N}_{b}(\mathbf{A})} P_{n}(\mathbf{A}) \overline{\varsigma_{n}^{n_{q}}}\right]\right)\right.\right. \\
& \left.\left.-\log \left(\prod_{n_{q} \in \mathcal{N}_{q}}\left[\sigma_{z}^{2}+\sum_{n \in \mathcal{N}_{b}(\mathbf{A}) \cap \overline{\mathcal{N}_{q}}} P_{n}(\mathbf{A}) \overline{\varsigma_{n}^{n_{q}}}\right]\right)\right]\right\}
\end{aligned}
$$

This concludes the derivation.

\subsection{Time Complexity Analysis: Simulation vs Closed form}

Algorithmic efficiency is computed using complexity analysis. Using the analytical derivation, the closed form representation of $\mathbb{E}\left[R_{q}(\mathbf{A})\right]$ over all $q$ is given by (19). Average of per cell sum rate is evaluated using 1000 Monte Carlo based iterations. These represent random fading and user distribution snaps. A for loop is conventionally used to average over these states. Figure 3 shows that simulation based analysis has complexity to the order of $n^{2}$. This explains the increasing gradient with increasing cells per cluster, $\left|\mathcal{N}_{q}\right|$ for simulated sum rate in Figure 3. For the closed form representation, these loops are no longer needed as the fading states are approximated using the law of large numbers. Further, Figure 3 shows that the order of complexity increases logarithmically in the order non-cooperative (NC-CMAC), cluster-cooperative (CC-CMAC) and full-cooperative (FC-CMAC) cellular MAC. 
$\bar{R}_{\pi(o), q}(\mathbf{A})=\tilde{\bar{R}}_{\pi(o), q}(\mathbf{A})-\sum_{i=1}^{o-1} \bar{R}_{\pi(i), q}(\mathbf{A})$

where,

$\tilde{\bar{R}}_{\pi(o), q}(\mathbf{A})=\left[\log \left(\prod_{m \in \mathcal{N}_{q}}\left[\sigma_{z}^{2}+\sum_{n \in \mathcal{N}_{b}(\mathbf{A})} P_{n, b}(\mathbf{A}) \overline{\varsigma_{n}^{m}}\right]\right)-\log \left(\prod_{m \in \mathcal{N}_{q}}\left[\sigma_{z}^{2}+\sum_{\substack{n \in \mathcal{N}_{\pi(o), b}^{+} \\ 0 \in \mathcal{N}_{q}}} P_{n, b}(\mathbf{A}) \overline{\varsigma_{n}^{m}}\right]\right)\right]$

This can be explained due to the reduced search space from FC-CMAC $\left(2^{B N}\right)$ to CC-CMAC $\left(2^{B\left|\mathcal{N}_{q}\right|}\right)$. This gives another motivation for choosing CC-CMAC which can be implemented for a range of practically realizable receivers in an AP cooperation based cellular system framework.

Figure 6 shows that the average Monte Carlo based simulation matches closely to the closed form representation for the 3 different Cellular MAC models. This justifies use of closed form representation in subsequent analysis.

\subsection{Derivation of closed form cell based QoS balancing func- tion}

We further perform SIC [14] on the cells within each cluster such that the sum rate of users within a cluster can be evaluated. In order to decode the cell based ordering from the cluster based sum rate, we need to first consider closed form of system wide sum rate of all cells represented by $\bar{R}(\mathbf{A})$ (19) from [12].

We consider MMSE-SIC detection[14] on the received signal at $q^{\text {th }}$ cluster (i.e. $\left.R_{q}(\mathbf{A})\right)$ with decoding ordered as follows: $1,2,3, \ldots, \frac{N}{Q}$. For a given bin allocation, $b$, we first detect the signal from cell 1 treating the signals from all the other cells within the cluster as interference, and then subtract the contribution of this interference from this signal. The detection process is then repeated for cell with index 2 up till index $\frac{N}{Q}$. Here it is assumed that $\left|\frac{N}{Q}\right|=7$. Defining $\pi(n)$ as the permutation of cells in set $\mathcal{N}_{q}$ with $\pi(1)$ being the first decoded cell and $\pi(7)$ as the last decoded cell. Generalizing for cell decoding order $\pi$ we have per cell sum rate for $o^{\text {th }}$ cell transmitting over $b^{\text {th }}$ bin in $q^{\text {th }}$ cluster defined as in (20).

Formulating (20), $\bar{R}_{\pi(o), q}$ (A) defines the signal from all cluster users transmitting over bin, b. $\tilde{\bar{R}}_{\pi(o), q}(\mathbf{A})$ defines the interference from users transmitting in cluster different from the reference cluster as well as signals from users in cells within the same cluster but not decoded. We further decode the per cell sum rate for $q^{\text {th }}$ cluster by deriving (21) from (20), where $\sigma_{z}^{2}$ is defined previously and $\mathcal{N}_{\pi(o), b}^{+}=\mathcal{N}_{b}(\mathbf{A}) \cap \overline{\mathcal{N}_{q}} \cup \mathcal{N}_{\pi(o)}^{+}$. Here the non-decoded cells within the cluster, are represented by the set, $\mathcal{N}_{\pi(o)}^{+}=\left\{\pi(o+1), \ldots, \pi\left(\frac{N}{Q}\right)\right\}$.

Lemma 1. For CC-CMAC using MMSE-SIC, the sum rate for $o^{\text {th }}$ cell in the $q^{\text {th }}$ cluster, has an ergodic sum rate given by:

$$
\bar{R}_{\pi(o), q}(\mathbf{A})=\left\{\begin{array}{lr}
\left.f\left(\mathcal{N}_{b}(\mathbf{A})\right)\right)-f\left(\mathcal{N}_{\pi(1), b}^{+}\right) & \text {if o }=\pi(1) \\
f\left(\mathcal{N}_{\pi(o-1), b}^{+}\right)-f\left(\mathcal{N}_{\pi(o), b}^{+}\right) & \text {otherwise } \\
\forall o \in \mathcal{N}_{q} &
\end{array}\right.
$$

where, for simplicity it is assumed that $f\left(\mathcal{N}_{b}(\mathbf{A})\right)=\log \left(\prod_{m \in \mathcal{N}_{q}}\left[\sigma_{z}^{2}+\sum_{n \in \mathcal{N}_{b}} P_{n, b}(\mathbf{A}) \overline{\varsigma_{n}^{m}}\right]\right) \quad \&$ 
$f\left(\mathcal{N}_{\pi(o), b}^{+}\right)=\log \left(\prod_{m \in \mathcal{N}_{q}}\left[\sigma_{z}^{2}+\sum_{\substack{n \in \mathcal{N}_{\pi(o), b}^{+} \\ o \in \mathcal{N}_{q}}} P_{n, b}(\mathbf{A}) \overline{\varsigma_{n}^{m}}\right]\right)$ and that $\frac{1}{B} \sum_{b=1}^{B}($.$) can be ig-$ nored from RHS of (21) for simplicity.

Proof. Here we attempt to derive (21) from (20). For $o=1$, and from (20), it is easy to show the sum rate for first decoded cell as :

$\bar{R}_{\pi(1), q}(\mathbf{A})=\tilde{\bar{R}}_{\pi(1), q}(\mathbf{A})=f\left(\mathcal{N}_{b}(\mathbf{A})\right)-f\left(\mathcal{N}_{\pi(1), b}^{+}\right)$

Similarly, for $o=2$,

$$
\begin{aligned}
& \bar{R}_{\pi(2), q}(\mathbf{A})=\tilde{\bar{R}}_{\pi(2), q}(\mathbf{A})-\bar{R}_{\pi(1), q}(\mathbf{A}) \\
& =f\left(\mathcal{N}_{b}(\mathbf{A})\right)-f\left(\mathcal{N}_{\pi(2), b}^{+}\right)-\left[f\left(\mathcal{N}_{b}(\mathbf{A})\right)-f\left(\mathcal{N}_{\pi(1), b}^{+}\right)\right] \\
& =\log \left(\prod_{m \in \mathcal{N}_{q}}\left[\sigma_{z}^{2}+\sum_{\substack{n \in \mathcal{N}_{\pi(1), b}^{+} \\
o \in \mathcal{N}_{q}}} P_{n, b}(\mathbf{A}) \overline{\varsigma_{n}^{m}}\right]\right) \\
& -\log \left(\prod_{m \in \mathcal{N}_{q}}\left[\sigma_{z}^{2}+\sum_{\substack{n \in \mathcal{N}_{\pi(2), b}^{+} \\
o \in \mathcal{N}_{q}}} P_{n, b}(\mathbf{A}) \overline{\varsigma_{n}^{m}}\right]\right)
\end{aligned}
$$

Similarly for $o=\frac{N}{Q}$,

$$
\begin{aligned}
& \left.R_{\pi\left(\frac{N}{Q}\right), q}(\mathbf{A})=\tilde{\bar{R}}_{\pi\left(\left[\frac{N}{Q}\right]\right), q}(\mathbf{A})-\bar{R}_{\pi\left(\left[\frac{N}{Q}\right]-1\right), q}(\mathbf{A})\right) \\
& =\log \left(\prod_{m \in \mathcal{N}_{q}}\left[\sigma_{z}^{2}+\sum_{\substack{n \in \mathcal{N}_{\pi\left(\left[\frac{N}{Q}\right]-1\right), b}^{+} \\
o \in \mathcal{N}_{q}}} P_{n, b}(\mathbf{A}) \overline{\varsigma_{n}^{m}}\right]\right) \\
& -\log \left(\prod_{m \in \mathcal{N}_{q}}\left[\sigma_{z}^{2}+\sum_{\substack{n \in \mathcal{N}_{\left.\pi\left(\left[\frac{N}{Q}\right]\right]\right), b}^{+} \\
o \in \mathcal{N}_{q}}} P_{n, b}(\mathbf{A}) \overline{\varsigma_{n}^{m}}\right]\right.
\end{aligned}
$$

Generalizing the above results by replacing cell indices with global variable $o$, one can get second part of (21). This sums up the proof.

\section{$5 \quad$ NP Hardness for Bin Allocation Problem (BAP) in CC-CMAC}

TThe aim of the BAP in fixed CC-CMAC is to assign bin resources to multiple cells such as to maximize QoS balancing function. In this section,it is assumed that QoS balancing function maximizes sum rate of all users. The sum rate maximization problem is a subset of the general QoS balancing function (which is a function of cell based sum rate).The well studied MI-FAP is mapped from literature [16] to the BAP Problem as per following definition.

Definition 1 (BAP using Multiple Bin for CC-CMAC). The achievable sum rate due to transmissions from users in the $Q$ clusters using $B$ bins, and received by the APs in the $Q$ clusters is defined as in (19).

Theorem 1. Solution to the BAP using Multiple Bin for CC-CMAC with $Q>>2$ is NP Hard. 
Proof. This section will highlight important steps which are detailed in [11]. Consider the clustering problem as the communication theoretic analogy of graph partitioning problem. This is defined as the division of total vertices, $\mathcal{V}$ into disjoint sets represented by $\mathcal{V}_{q}$ such that the number of edges whose end points are in $Q-1$ different vertices subsets are minimized.

Vertices of graph play role of transmitters in CC-CMAC and edges model point to point link between nodes. Define a cut across sets within and without cluster $q$ and define the conductance of cut as a measure of cluster quality. This is considered analogous to system efficiency of CC-CMAC.

$\mathcal{U}_{q}$ is the set of transmitters in $q^{\text {th }}$ cluster. Since users are colocated with receivers, $\left|\mathcal{U}_{q}\right|=\left|\mathcal{V}_{q}\right|$. These terms are used interchangeably denoting transmission and reception nodes in graph theoretic framework. Sum rate contribution due to transmission from users within $q^{\text {th }}$ cluster is denoted by, $R_{a}$ which is as follows,

$$
R_{a}=\log \left(\prod_{v \in \mathcal{V}_{q}}\left[\sigma_{z}^{2}+\sum_{u \in \mathcal{U}_{q}} P_{u} \overline{\varsigma_{u}^{v}}\right]\right)
$$

where, $\sigma_{z}^{2}$ is the noise variance and $P_{u}$ is the maximum transmit power for users in $u$. Similarly, sum rate contribution due to transmission from users outside $q^{\text {th }}$ cluster is denoted by, $R_{b}$. Hence,

$$
R_{b}=\log \left(\prod_{v \in \mathcal{V}_{q}}\left[\sigma_{z}^{2}+\sum_{u \notin \mathcal{U}_{q}} P_{u} \overline{\varsigma_{u}^{v}}\right]\right)
$$

Both $R_{a}$ and $R_{b}$ can be regarded as specific instances of $\bar{R}(\mathbf{A})$ (19). These can be evaluated from (19) using MMSE-SIC techniques [14].

\subsection{Active receivers in single cluster $\left(\left|\mathcal{V}_{q}\right|=\left|\mathcal{N}_{q}\right|\right)$}

Consider $\mathcal{W}$ as the set of all edges. $|\mathcal{W}|$ increases linearly with $|\hat{\mathcal{U}}|$. Further denote $\mathcal{W}_{q}^{\text {intra }} \&$ as the set of graph edges with both endpoints lie within $q^{\text {th }}$ cluster. Similarly. $\mathcal{W}_{q}^{\text {inter }}$ is the set of edges with one endpoint in and one out of $q^{\text {th }}$ cluster. Further, $\mathcal{W}_{q}=\mathcal{W}_{q}^{\text {intra }} \cup \mathcal{W}_{q}^{\text {inter }}$. Hence the following mapping function can be formulated,

$$
\begin{aligned}
& R_{a}(\hat{\mathcal{U}}) \longmapsto\left|\mathcal{W}_{q}^{\text {intra }}\right| \\
& R_{b}(\hat{\mathcal{U}}) \longmapsto\left|\mathcal{W}_{q}^{\text {inter }}\right|
\end{aligned}
$$

where $\longmapsto$ refers to the mapping between number of interfering edges to the sum rate contribution due to transmission from users within $R_{a}(\hat{\mathcal{U}})$ and outside $R_{b}(\hat{\mathcal{U}})$ the cluster.

\subsection{Active receivers in multiple clusters $\left(\left|\mathcal{V}_{q}\right|>\left|\mathcal{N}_{q}\right|\right)$}

Assuming identical clusters, and using $\min \left(\left|\overline{\mathcal{W}_{q}^{\text {intra }}}\right|,\left|\mathcal{W}_{q}\right|\right)=\left|\mathcal{W}_{q}\right|$.

Also as in [17], take subset $\mathcal{S}$ of $\mathcal{V}$ and define a cut $(\mathcal{S}, \mathcal{V} / \mathcal{S})$. Here, for BAP in CC-CMAC, the cut is represented by $\left(\mathcal{N}_{q}, \overline{\mathcal{N}_{q}}\right)$ where $\mathcal{N}_{q} \subseteq \mathcal{N}$ and $\mathcal{N}$ is the set of all cells within the system. Applying concept of graph clustering from [17], the following can be deduced:

$$
\begin{aligned}
\phi\left(\mathcal{N}_{q}\right) & =\frac{\left|\mathcal{W}_{q}^{\text {inter }}\right|}{\min \left(\left|\overline{\mathcal{W}_{q}^{\text {intra }}}\right|,\left|\mathcal{W}_{q}\right|\right)} \\
& =\frac{\left|\mathcal{W}_{q}^{\text {inter }}\right|}{\left|\mathcal{W}_{q}\right|}
\end{aligned}
$$


From [17], it is known that conductance of graph cluster, $q$ i.e. $\phi\left(\mathcal{N}_{q}\right)$ will be smallest conductance within that cluster. Further the conductance of graph is minimum conductance over all possible clusters, $q$. Applying to CC-CMAC this would imply a spectral efficiency measure over all clusters $Q$. Hence,

$$
\begin{aligned}
\phi(G) & =\min _{\mathcal{N}_{q} \subseteq \mathcal{N} ; \forall q} \phi\left(\mathcal{N}_{q}\right) \\
& =\max _{\mathcal{N}_{q} \subseteq \mathcal{N} ; \forall q}\left(\frac{1}{\phi\left(\mathcal{N}_{q}\right)}\right) \\
& =\max _{\forall q}\left(\frac{R_{a}+R_{b}}{R_{b}}\right) \\
& =\max _{\forall q \in\{1,2\}}\left(\frac{\log \left(\prod_{v_{1} \in \mathcal{V}_{q}}\left[\sigma_{z}^{2}+\sum_{n \in \mathcal{N}} P_{n} \overline{\varsigma_{n}^{v_{1}}}\right]\right)}{\log \left(\prod_{v_{1} \in \mathcal{V}_{q}}\left[\sigma_{z}^{2}+\sum_{n \in \mathcal{N} \cap \overline{\mathcal{N}_{q}}} P_{n} \overline{\varsigma_{n}^{v_{1}}}\right]\right)}\right)
\end{aligned}
$$

(28) follows from (27). In terms of rate contribution as a measure of conductance, (28) can be expanded to represent (29).

\subsection{Multiple bin allocation for reception at receivers within multiple clusters}

In a multiple cluster multiple bin BAP, (29) can be extended from single bin to multiple bins formation i.e. from $B=1$ to $B>>1$ and average over a bin. The number of receivers $\left|\mathcal{V}_{q}\right|$ increases for $Q>>2$. This suits the requirement of 7 clusters and 7 cells per cluster formation as implemented for Definition 1. Being a subset of BAP general problem, if (27) is proved NP Hard, then the generalized BAP for CC-CMAC is also NP Hard. From [17] and [16], solving (27) is proved to be NP Hard. Here, (29) is equivalent to (27).Hence (29) is also proven as NP Hard.

Since BAP for Multiple Bin allocation in CC-CMAC is a more general case of (29), Definition 1 is also NP Hard. QoS balancing function is a function of sum rate i.e. depends on (19) and therefore Definition 1. Optimizing the network QoS balancing function problem as defined in Section 4 is therefore an NP Hard problem.

\section{GA based implementation of QoS Balancing func- tion in CC-CMAC}

GA is a powerful optimization tool widely used in solving channel allocation problems including dynamic channel allocation $[18,19]$ and bin allocation in Cellular $\mathrm{MAC}[12]$.

To optimize efficiency of allocation for CC-CMAC users, (5) is used as an objective function to a modified GA. The allocation is optimized over a number of generations. In each generation, the allocation matrix encoded onto a number of chromosome strings is evaluated. The crossover technique is selected such that the crossover point separates allocation for different users. Mutation is implemented by flipping the alleles [20] to any of the $2^{V}-1$ alternate power states. The termination criteria is determined by the number of generations over which the efficiency is near constant. These are summarized in Figure 4 and detailed as in [11]. 


\subsection{GA Implementation}

The modification of GA with respect to generic string encoding, using fitness function, crossover technique and mutation as depicted in Figure ref takes place as follows:

\subsubsection{Encoding of Bin Allocation Matrix}

The method of representation (encoding) for a chromosome has a major impact on GA's performance. Binary string encoding is selected since it helps to select from a large number of possibilities using few trials. Compared to a binary string of 5 a binary string of 10 has 32 times larger schemata. The string length $(B \times$ $N$ ) represents total number of genes. Each gene represents allocation of a specific bin to a specific cell for CC-CMAC. Further, binary strings were chosen for simplicity of operation [20]. Similarly in this work a 0 signifies no allocation whereas a non zero allele indicates an allocation.

\subsubsection{Block sized Crossover}

This type of crossover puts restriction on the location of the plane of crossover. This is required since the allocation on either side of the plane should belong to different cells. GA would otherwise descend to premature convergence [11]. This scheme is depicted in Figure 5. Here Parent 1 and Parent 2 pass their characteristics to Child 1 and Child 2. This is shown by a direct mapping from Parent 1 to Child 1 and Parent 2 to Child 2. Beyond the plane of crossover, genes are swapped for the remaining chromosome such that Child 1 receives Parent 2's genes and vice versa. The above is verified after creating test points in the Matlab simulation. Secondly, content of the chromosome is verified before and after the crossover operation. Moreover, the bins allocated to each user are summed up. This should be the same before and after the crossover operation.

\subsubsection{Elitism}

Fitness function is a non negative figure of merit [21] used to quantify the 'best fit' amongst the population. Survival of the fittest translates to discarding the chromosomes which are unfit. In the bin allocation problem, bins are allocated such that they maximize sum rate. This is done by reshaping encoded matrix from the chromosome string to a bin allocation table. This allocation matrix denotes the input matrix for (19). After GA has reached maximum generations, the encoded matrix is converted back to chromosome strings.

As can be observed, the fitness function (2) is a summation of user rates in all $Q$ clusters. In each cluster, the sum rate is of the form $R_{q}(\mathbf{A})=\frac{1}{B} \sum_{b=1}^{B} \log \operatorname{det}\left\{\frac{\mathbf{I}}{\mathbf{J}}\right\}$. Here $B$ is constant over a given run and depends on allocation $\mathbf{A}$ dimensions. I represents the received signal strength as observed by the receivers in $q^{\text {th }}$ cluster, from all transmitters in the system (i.e. inside and outside the $q^{\text {th }}$ cluster) and $\mathbf{J}$ is received signal strength of the transmissions from outside the cluster $q$. The set of conditions under which $\mathbf{J}$ is minimized and $\mathbf{I}$ is maximized, hence determines the 'best fit' solution to the GA under study.

\subsubsection{Selection}

The concept of elitism was implemented with a modified version of De Jong's elitist model [22]; the best member of current population is forced to become member of the next population. This helps maximize sum rate over all generations. The fitter the parents, the higher the chances that they are selected. 


\subsubsection{Crossover}

Crossover is the main genetic operator which preserves inherit characteristics from each parent using a "cut-catenate" technique. Single point crossover is the simplest of all crossover techniques. [18]; hence it is adopted in this analysis. However, Figure 5 shows that point of crossover cannot bisect bins allocated to a single user (shaded group of 3 bins). If that is the case, it will result in the algorithm converging prematurely.

The sort of single point crossover used depends on restriction on the location of the plane of crossover. This is required since the allocation on either side of the plane should belong to different cells. GA would otherwise descend to premature convergence [11]. This scheme is depicted in Figure 5. Here Parent 1 and Parent 2 pass their characteristics to Child 1 and Child 2. This is shown by a direct mapping from Parent 1 to Child 1 and Parent 2 to Child 2. Beyond the plane of crossover, genes are swapped for the remaining chromosome such that Child 1 receives Parent 2 's genes and vice versa. The above is verified after creating test points in the Matlab simulation. Secondly, content of the chromosome is verified before and after the crossover operation. Moreover, the bins allocated to each user are summed up. This should be the same before and after the crossover operation. Crossover takes place with a set probability, $\mathcal{P}_{c}$. If $\mathcal{P}_{c}=0$, then the new chromosome population is a copy of the old. If $\mathcal{P}_{c}=1.0$ all the offspring are made by crossover. In our analysis, $\mathcal{P}_{c}$ is in the range of $0.75-0.90$.

\subsubsection{Variable Mutation}

Mutation is a genetic operator which transforms individual chromosomes by randomly changing allele (inverting bit positions) of some genes. The operation is carried out on the allocated bins and varied as per sum rate gradient in order to respond the random nature of user positioning . Mutation takes place with a very low bit probability, to prevent the GA from becoming a random search operation. Probability of mutation on a bit, $\mathcal{P}_{m}$ is hence in the range $0.0001-0.01$.

\subsubsection{Termination Condition}

The termination condition specifies whether the algorithm needs to continue searching or stop. When no further bin allocation maximizes sum rate and the population has converged, the GA terminates. In this implementation, similar fitness values over consecutive generation indices satisfies termination condition. The GA will terminate if the fitness value is consistent for the last 10 consecutive generations.

A total of 16 simulations were carried out. The total number of users $K$, transmitting in each cell was modified such that:

- 4 sets of experiment were carried out using varying population size, $M$ for $K>100$

- 4 sets of experiment with different $\operatorname{crossovers}\left(M^{-1} \leq \mathcal{P}_{c} \leq M\right), K>100$

- 4 sets of experiment were carried out with varying $M, K<100$

- 4 sets of experiment with different $\operatorname{crossovers}\left(M^{-1} \leq \mathcal{P}_{c} \leq M\right), K<100$

The above parameters were optimized by tuning to gain stability within the experiment (consistency in values over the last 10 generations), and the efficiency achieved (difference in sum rates between first and last completed generation cycle).

To sum up, the bin allocation is optimized over a number of generations. In each generation, the allocation matrix is encoded onto a number of chromosome strings 
and each string is evaluated against an objective criteria (optimization of resource allocation). The crossover technique is selected such that the crossover point separates allocation for different users. Mutation is implemented by flipping the alleles [20] to any of the $2^{V}-1$ alternate power states. Here $V$ is the total number of bits encoded for bin allocation to every user. The termination criteria is determined by the number of generations over which the efficiency is near constant. These are summarized in Figure 4 and detailed as in [11].

\section{Results \& Discussion}

Figure 6 shows the effect of $V$ and $B$ on sum rate for CC-CMAC. For high density APs, the efficiency of GA optimized allocation in CC-CMAC approaches the upper bound. For less dense systems, the difference between GA optimized and full reuse allocation schemes reduces to nearly 0 . The given parameters make GA suitable for dense urban centres.

\subsection{Impact of fairness on QoS : max-min fairness}

The max-min fairness is a tractable and flexible fairness model that helps to compare a range of fairness conditions. It is known that rate region for (21) is not convex in general. Since class of utilities depending on per cell sum rate should have a convex formulation as their input, further conditions are required to optimize the fairness formulation using cell based sum rate.

Using function $h(x)$ of the form $\left(e^{x}-1\right)^{-1}$ satisfies the conditions for maximizing minimum sum rate contribution of users in a cell such that any further increase will likely to decrease sum rate allocated to higher rate cell users [11]. Lemma : If $h(x)$ is differentiable increasing negative \& concave function than given $x \geq 0$, the solution of $U_{\gamma}(h(x))$ approaches max - min fair vector for $\gamma \rightarrow 10$.

From definition of log-concave, we know that a function is $\log$-concave if $\log f$ is concave. We know that cumulative Gaussian Proability Function is log - concave. Applying the same to the fairness formulation introduced, it can be shown that [11]:

$$
u_{\gamma}\left(R_{m, q}(\mathbf{A})\right)=\frac{-\left[h\left(R_{m, q}(\mathbf{A})\right)\right]^{-\gamma}}{\gamma} \max -\min \mathrm{F} .
$$

Here, $u_{\gamma}\left(R_{m, q}(\mathbf{A})\right)$ is the service balancing function for users in $m^{\text {th }}$ cell in $q^{\text {th }}$ cluster using fairness coefficient of $\gamma$. Simplifying, the following can be deduced

$$
u_{\gamma}\left(R_{m, q}(\mathbf{A})\right)=\frac{1}{\gamma}\left[\frac{-1}{\left(e^{\left(R_{m}, q(\mathbf{A})\right)}-1\right)}\right]^{\gamma} \max -\min \mathrm{F} .
$$

Based on the above, it is imperative to compare fairness with the system efficiency. Having a fair distribution of resources will reduce the numerator for $R$. However, the sum rate contribution of edge cells will increase since they now have more resources allocated to them. Since the number of edge cells (6) is far greater than the non edge cells(1), there is an increase in interference to receivers in adjacent clusters. This reduces the over all sum rate further. However, the CDF determines in 2 stages the effect of fairness and improvement of group performance (cell users) within a cluster.

- The $10^{t h}$ percentile is used to gauge performance of users in cell edge.

- The $90^{\text {th }}$ percentile is used to gauge performance of users in cell centre. 
Since the fairness described is strict and effects the edge cell users most, 10th percentile is generally regarded as the determining metric for qualifying edge cell users since their sum rate contribution is the least [11].

For $D=1000 \mathrm{~m}$, sum rate contribution due to hard fairness optimized QoS balancing function is shown in Figure 6 and the plot in Figure 8. For nominally dense APs, the sum rate is reduced by about $20 \%$. However, the minimum rate of disadvantaged users is increased by $10 \%$. At the 10 th-percentile reference, sum rate for MMF optimized allocation is 5.1 as compared to 4.7 for maximize sum rate. Hence $10 \%$ users have rates which are atleast $0.5 \mathrm{bps} / \mathrm{Hz} /$ cell higher than that due to QoS balancing function without fairness. This gap is further increased if the cross over points are varied along with the poulation size. That is the cross over points are not predetermined.

In lower percentile CDFs, lower sum rate contributors are less prone to interference from other low sum rate contributing cells in alternate clusters. This is not the same for higher cell sum rate contributors which are more prone to interference. Using variable crossover points, the resource allocation becomes more flexible and scant resrouces are allocated to the very few users. This improves the situation as depicted for $10^{\text {th }}$ percentile users. The increasing sum rate contribution leads to interference which overshadows the advantage in flexible bin allocation due to variable corssover points. Hence the CDFs converges for percentiles greater than the median. Figure 7 shows that at ISD of $1000 \mathrm{~m}$, implementing MMF for higher coefficient results in greater contribution of per cell sum rate from $10^{t h}$ percentile users than JFI using the same fairness coefficient. The median for MMF is also more in line with the average per cell sum rate which is not afected by changing the degree of fairness $(\eta=2$ to $\eta=10)$. This explains the choice of MMF as fairness metric in the final evalaution of QoS Balancing function Figure 7.

\subsection{Analysis of GA Optimized Allocation Design}

\subsubsection{Signalling Analysis}

Dimensions of channel matrices reveal complexity requirement for cell pathloss feedback. This is used to measure the signalling overhead for different Cellular MAC. For FC-CMAC, average cell based pathloss information from each of $N$ user groups to the $N$ APs in system are represented by the $N^{2}$ bits for transferring 1 bit per cell. Due to power granularity each cell information now requires $V$ bits for transmission. Hence the total signalling requirement is $V N^{2}$ bits for FC-CMAC. It is worth noting that $N=Q\left|\mathcal{N}_{q}\right|$ for any Cellular MAC. For CC-CMAC, the signalling requirement is for $V Q\left|\mathcal{N}_{q}\right|^{2}$ bits per snapshot. CC-CMAC requires $Q$ times less signalling overhead. This is one of the motivations for choosing CC-CMAC.

\subsubsection{Complexity}

The search space for joint power and bin allocation depends on $B, V$, and $N$ as per the following relationship $2^{N V B}$. Hence for fixed $N$ and $V$, the complexity rises to the order of $2^{B}$. The complexity is hence a function of the chromosome length (total bins allocated). As a motivation to reduced chromosome length, it is possible to reduce $B$ while at the same time increase $V$ such as not to affect the increase in sum rate. This approach makes the algorithm less computationally intensive and GA becomes feasible for modern OMC-Rs and centralized frequency planners as in Figure 2. 


\section{Conclusions}

A novel QoS balancing framework for CC-CMAC is proposed in this work. The aim is to increase spectral efficiency as well as achieve cell based fairness for given per user power constraint. This is implemented by deriving cell based QoS balancing function. Using analogy with graph conductance, QoS balancing problem is proved to be NP Hard. Using joint frequency and power granularity, this problem is formulated as an objective function input to a GA and compared for a range of ISDs and bin and power quantization states. Results show that maximizing sum rate CC-CMAC can help achieve the upper bound of the capacity region in highly dense AP scenario. Using max - min fairness in moderately dense AP scenarios, reduced sum rate (improvement over full reuse), can effect a slight increase in fairness. A practical centralized frequency planner for implementing modified GA is proposed. This framework has application for both HP and BE customers [23] alike. Current research work under way looks into tapered cellular architectures and extension to comply with further restriction as per a multi objective optimization design.

\section{References}

[1] A. D. Wyner, "Shannon-theoretic approach to a Gaussian cellular multipleaccess channel," IEEE Trans. Inf. Theory, vol. 40, no. 6, pp. 1713-1727, Nov. 1994.

[2] O. Somekh and S. Shamai, "Shannon-theoretic approach to a Gaussian cellular multiple-access channel with fading," IEEE Trans. Inf. Theory, vol. 46, no. 4, pp. 1401-1425, Jul. 2000.

[3] N. Letzepis and A. Grant, "Information capacity of multiple spot beam satellite channels," in Communications Theory Workshop, 2005. Proceedings. 6th Australian, 2-4 Feb. 2005, pp. 168-174.

[4] P. Marsch and G. Fettweis, "A Framework for Optimizing the Uplink Performance of Distributed Antenna Systems under a Constrained Backhaul," Proc. IEEE International Conference on Communications (ICC '07), pp. 975-979, Jun. 24-28, 2007.

[5] E. Aktas, J. Evans, and S. Hanly, "Distributed Decoding in a Cellular MultipleAccess Channel," IEEE Trans. Wireless Commun., vol. 7, no. 1, pp. 241-250, Jan. 2008.

[6] W. Choi and J. G. Andrews, "Downlink performance and capacity of distributed antenna systems in a multicell environment," IEEE Trans. Wireless Commun., vol. 6, no. 1, pp. 69-73, Jan. 2007.

[7] O. Somekh, B. M. Zaidel, and S. Shamai, "Sum rate characterization of joint multiple cell-site processing," IEEE Trans. Inf. Theory, vol. 53, no. 12, pp. 4473-4497, Dec. 2007.

[8] N. Levy and S. Shamai, "Clustered local decoding for wyner-type cellular models," IEEE Trans. Inf. Theory, vol. 55, no. 11, pp. 4967-4985, 2009.

[9] Z. Shen, J. G. Andrews, and B. L. Evans, "Adaptive resource allocation in multiuser OFDM systems with proportional rate constraints," IEEE Trans. Wireless Commun., vol. 4, no. 6, pp. 2726-2737, Nov. 2005. 
[10] A. Papadogiannis, D. Gesbert, and E. Hardouin, "A Dynamic Clustering Approach in Wireless Networks with Multi-Cell Cooperative Processing," Proc. IEEE International Conference on Communications (ICC '08), pp. 4033-4037, May 19-23, 2008.

[11] M. I. Majid, Frequency Planning for Clustered Jointly Processed Cellular MAC. PhD thesis, University of Surrey, Guildford, UK, 2010.

[12] M. I. Majid, M. A. Imran, and R. Hoshyar, "Optimization of uplink sumrate for bin based clustered cellular system using a genetic algorithm," Proc. 6th International Wireless Communications and Mobile Computing Conference (IWCMC '10), pp. 1016-1020, 2010.

[13] —_ "Cell based Fair Resource Allocation in Fixed Clustered Cellular Systems using a Genetic Algorithm," Proc. IEEE 21st International Symposium on Personal, Indoor and Mobile Radio Communications, (PIMRC 2010), 2010.

[14] D. Tse and P. Viswanath, Fundamentals of Wireless Communications. University Press, Cambridge, 2005.

[15] T. M. Cover and J. A. Thomas, Elements of Information Theory, Second ed. John Wiley \& Sons, 2006.

[16] G. Colombo and S. Allen, "A Decomposed Approach for the Minimum Interference Frequency Assignment," Linkage in Evolutionary Computation, pp. $389-417,2008$.

[17] R. Kannan, S. Vempala, and A. Vetta, "On Clusterings: Good, Bad and Spectral," J. ACM, vol. 51, no. 3, pp. 497-515, 2004.

[18] D. Thilakawardana, K. Moessner, and R. Tafazolli, "Darwinian approach for dynamic spectrum allocation in next generation systems," IET Communications, vol. 2, no. 6, pp. 827-836, Jul. 2008.

[19] G. Song and Y. Li, "Cross-layer optimization for OFDM wireless networks-part I: theoretical framework," IEEE Trans. Wireless Commun., vol. 4, no. 2, pp. 614-624, Mar. 2005.

[20] D. E. Goldberg, Genetic Algorithms in Search, Optimization and Machine Learning. Boston, MA, USA: Addison-Wesley Longman Publishing Co., Inc., 1989.

[21] M. Mitchell, An Introduction to Genetic Algrithms. MIT Press, 1998.

[22] K. T. K.F. Man and S. Kwong, Genetic Algorithms. Springer-Verlag, London, 2001.

[23] S. Bashar and Z. Ding, "Admission control and resource allocation in a heterogeneous OFDMA wireless network," IEEE Trans. Wireless Commun., vol. 8, no. 8, pp. 4200-4210, Aug. 2009. 


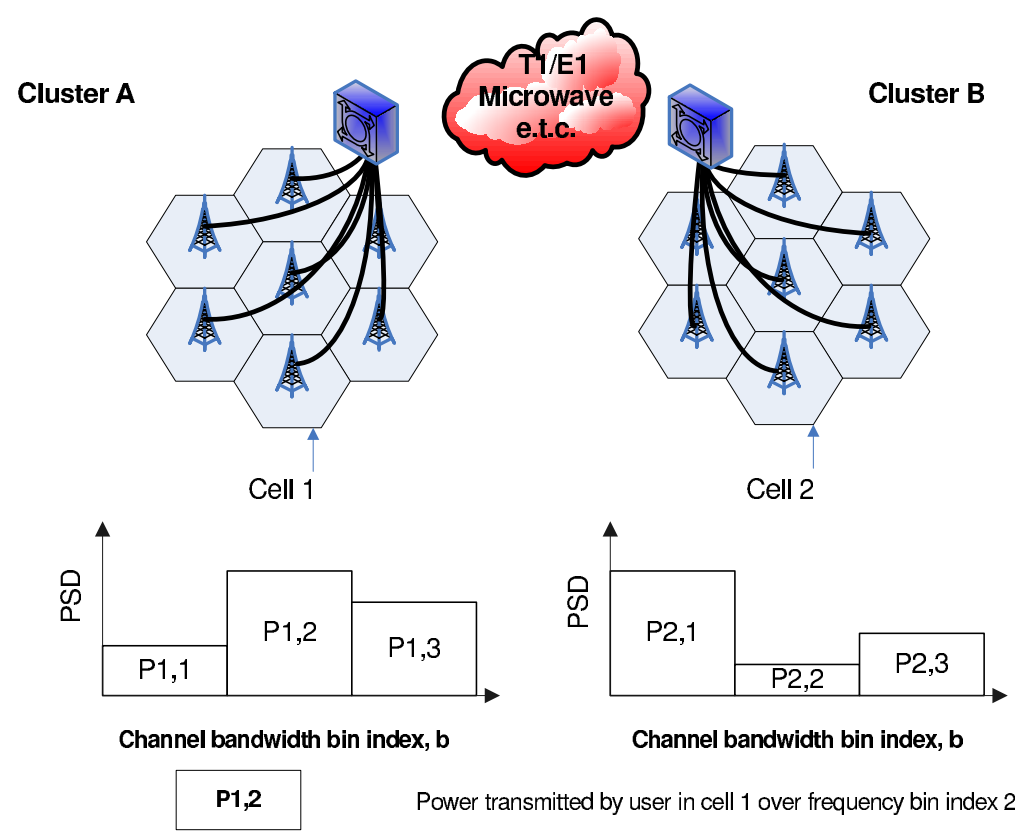

Figure 1: Demonstration of Uplink Transmission by users in cells from 2 adjacent clusters. Here the scenario is shown across 3 bins which are allocated power to cater interference in Fixed Cluster as per CC-CMAC. Here the backhaul is also shown. 


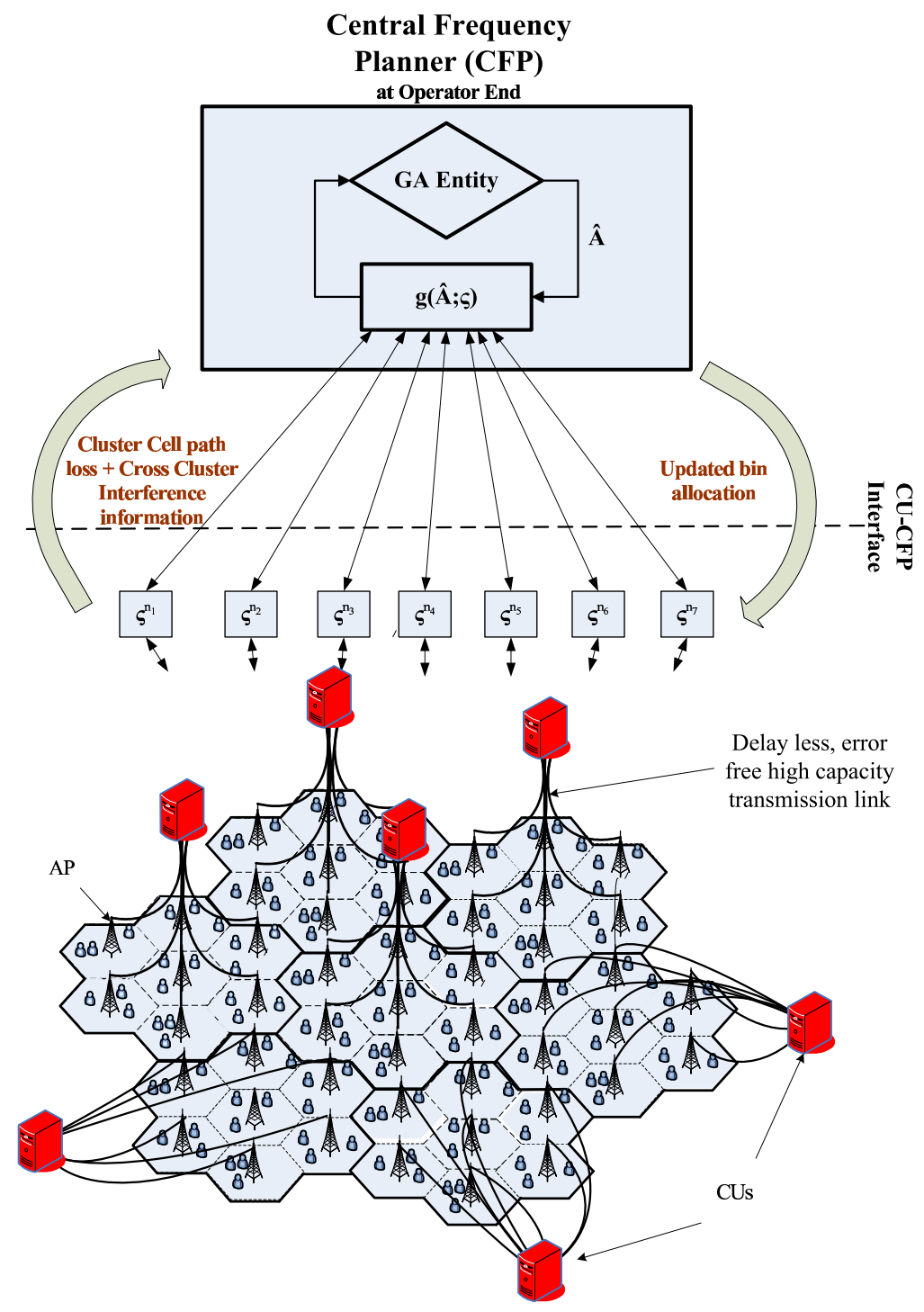

Figure 2: GA architecture as implemented for QoS balancing formulation for a CC-CMAC. 


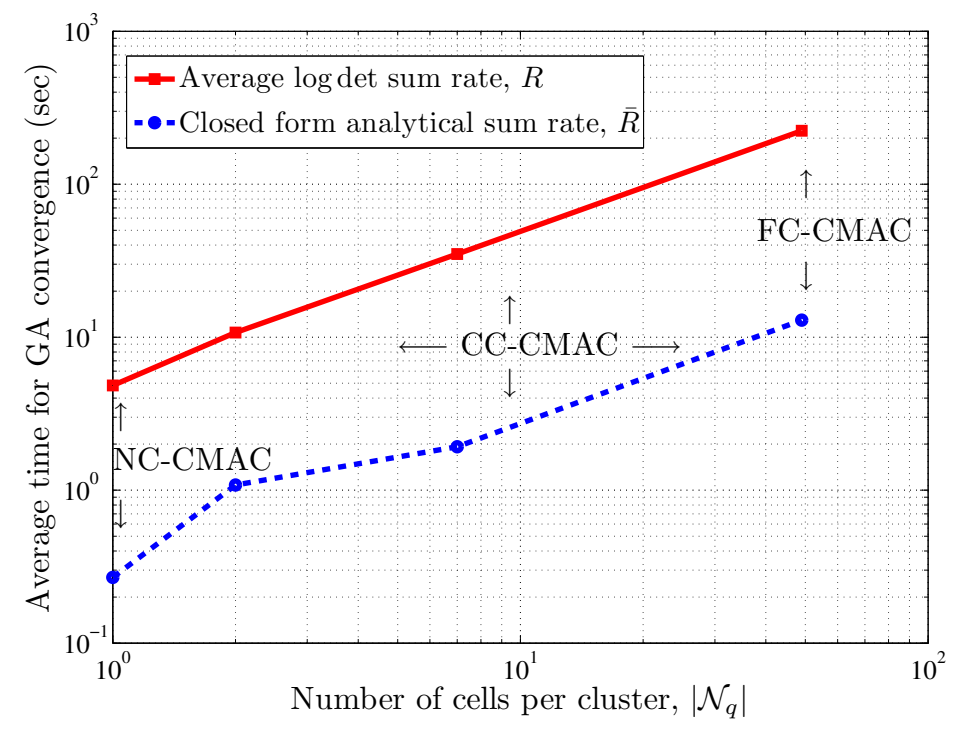

Figure 3: Time complexity comparison between $\mathbb{E}\left[R_{q}(\mathbf{A})\right] \forall q \& \bar{R}(19)$ as implemented using GA for uplink transmission undergoing flat fading and pathloss in bin based Cellular MAC design. Here $B=5, K=60$. For $\log \operatorname{det}$ simulation, $D$ is averaged over 1000 runs. For comparison the time complexity of NC-CMAC $(Q=49)$ and FC-CMAC $(Q=1)$ are also shown. 


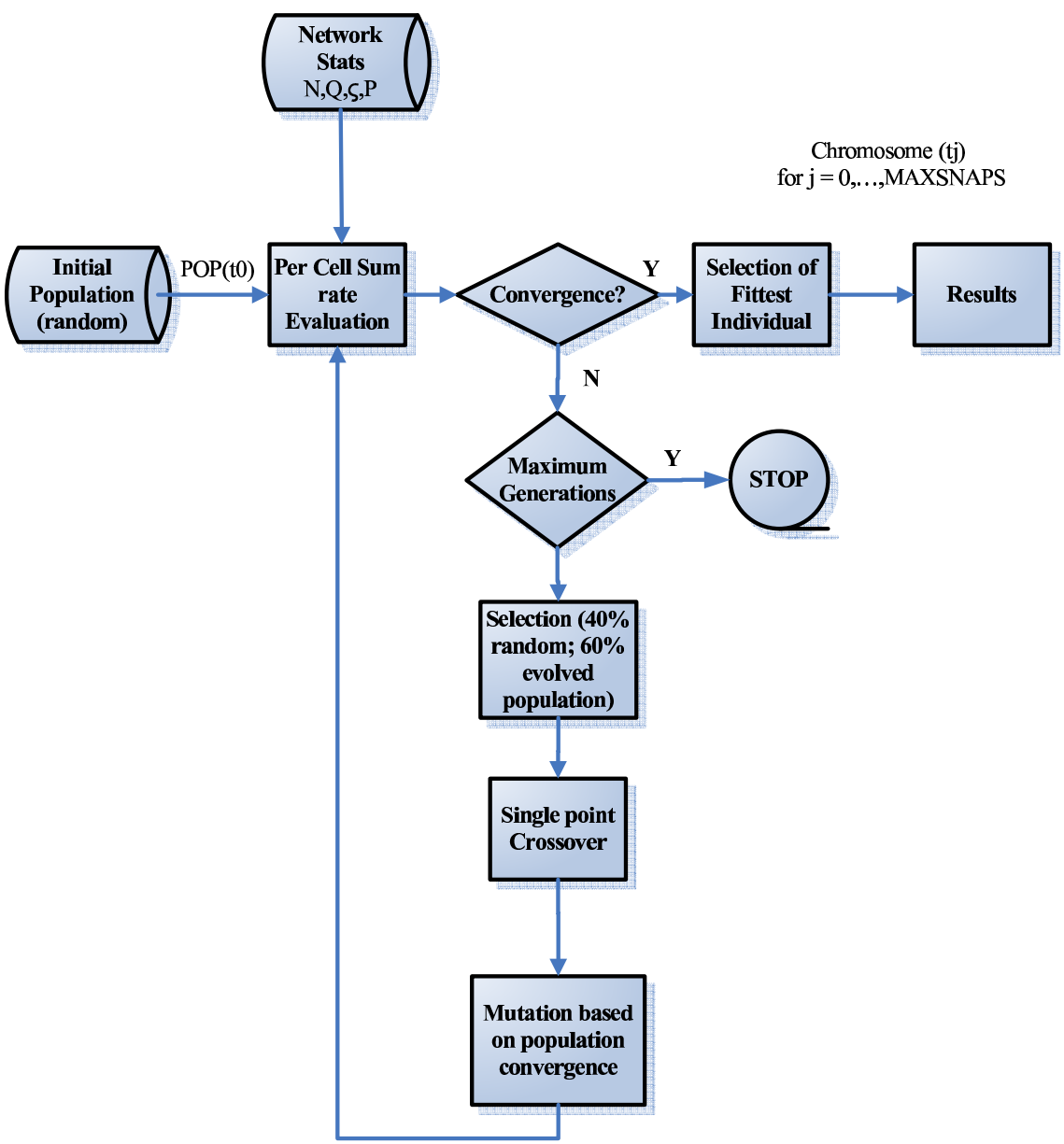

Figure 4: GA Flowchart for optimizing bin based allocation in CC-CMAC. POP(t0) represents the population at reference time, t0. MAXSNAPS is the maximum number of time snaps. 


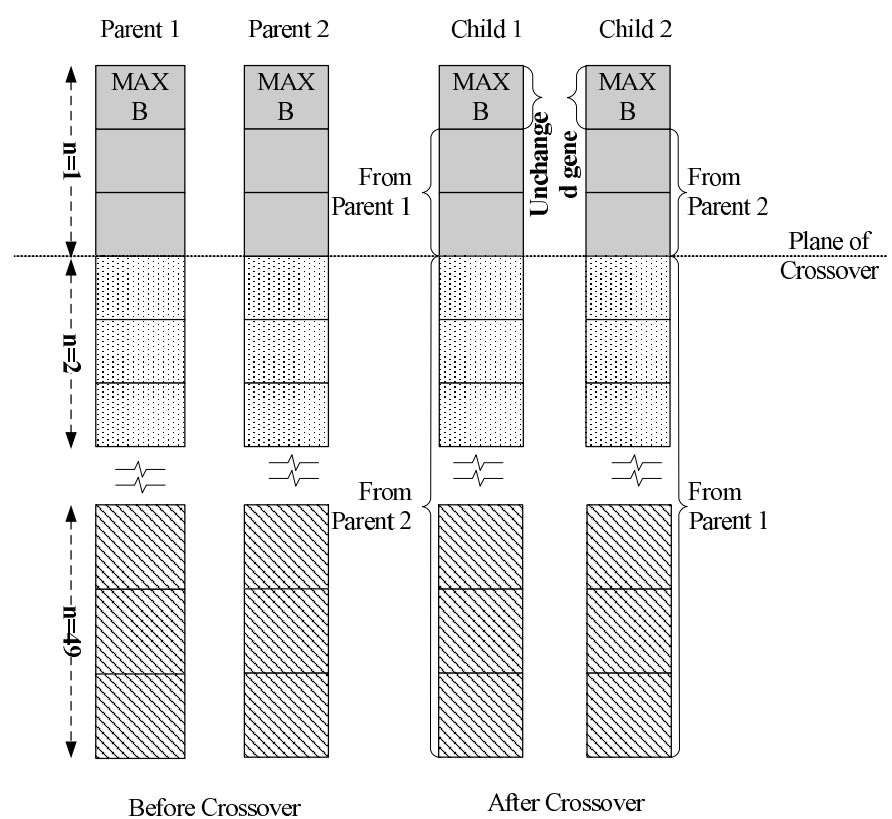

Figure 5: Modified GA : Block size crossover operation for a CC-CMAC.

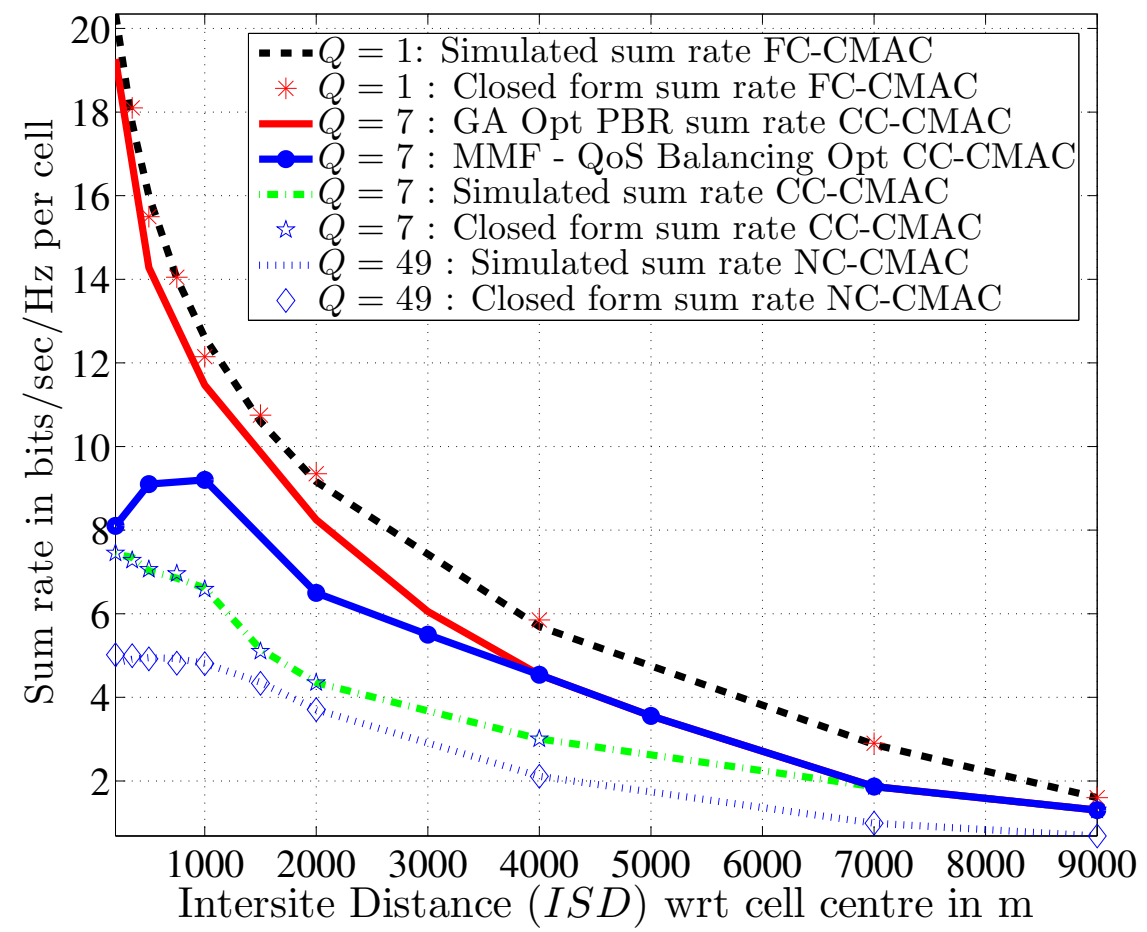

Figure 6: Effect of ISD on Per Cell Sum Rate due to GA optimized Partial bin Reuse $(\mathrm{PBR})$ and $\max -\min$ fairness via joint power and bin allocation. Here $V=5$, $B=10$, AP density is varied from ISD $=200$ to9000m, $N=49, Q=7, \eta=3.5$, $L_{o}=31.5 \mathrm{~dB}$, and $\sigma_{o}^{2}=16.9 \mathrm{dBm} / \mathrm{Hz}$ over $5 \mathrm{MHz}$ bandwidth. For comparison, full reuse as implemented on NC-CMAC $(Q=49)$ and FC-CMAC $(Q=1)$ are also shown. 


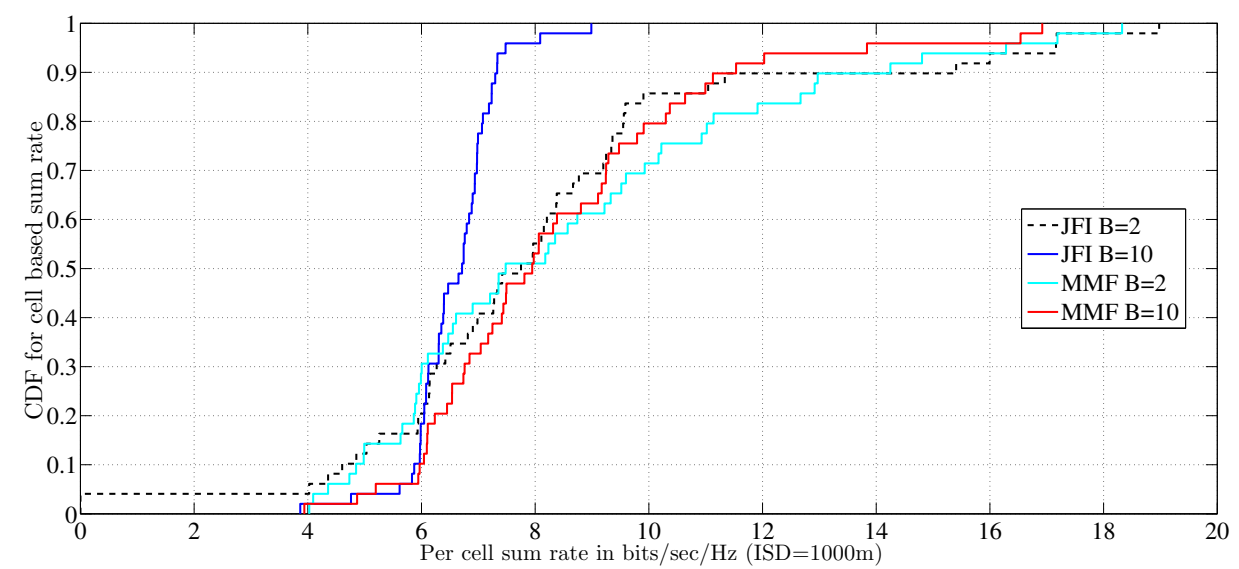

Figure 7: Comparison between fairness indices for Jains Fairness Index (Jain's Fairness Index) and max - min fairness (MMF). The per cell sum rate degrades for the Jain's Fairness Index for lower fairness coefficient and smaller number of bins. The MMF is said to exhibit better performance as its slope about median is simply a function of per cell sum rate when bin granularity is increased. Here for $B \geq 2$ MMF fares better than JFI which is a common metric for measuring fairness.

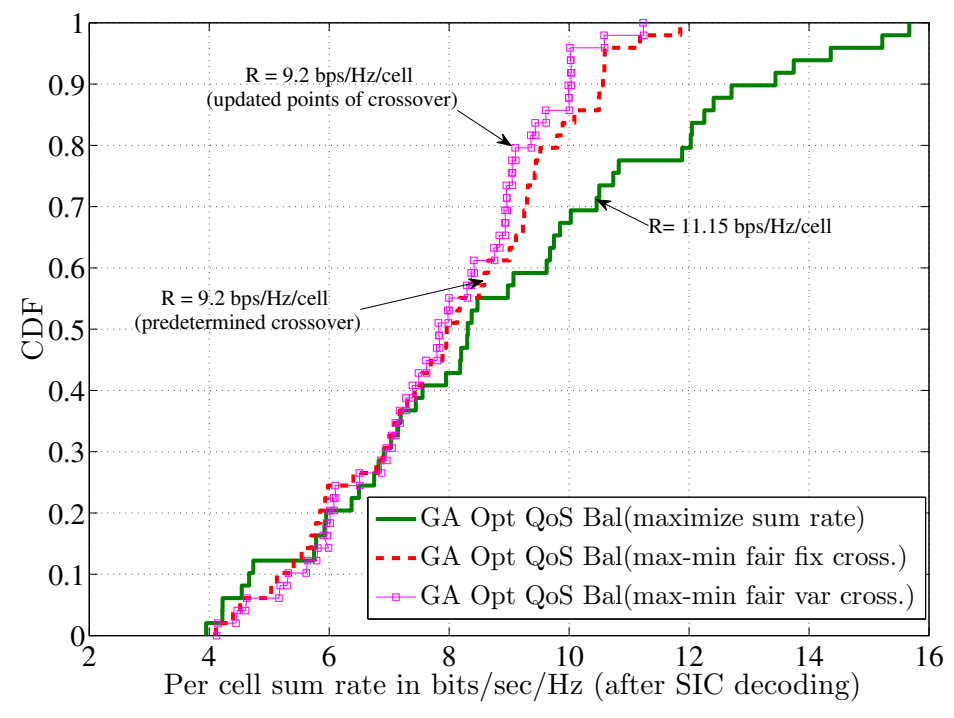

Figure 8: Effect of $\gamma$ on CDF of joint power and bin allocation. Here $V=5$, $B=10$, ISD $=1000, N=49, Q=7, \eta=3.5, L_{o}=31.5 \mathrm{~dB}$, and $\sigma_{o}^{2}=16.9$ $\mathrm{dBm} / \mathrm{Hz}$ over $5 \mathrm{MHz}$ bandwidth. For comparison the effect of block sized crosssover (both predetermined and variable) are shown. 


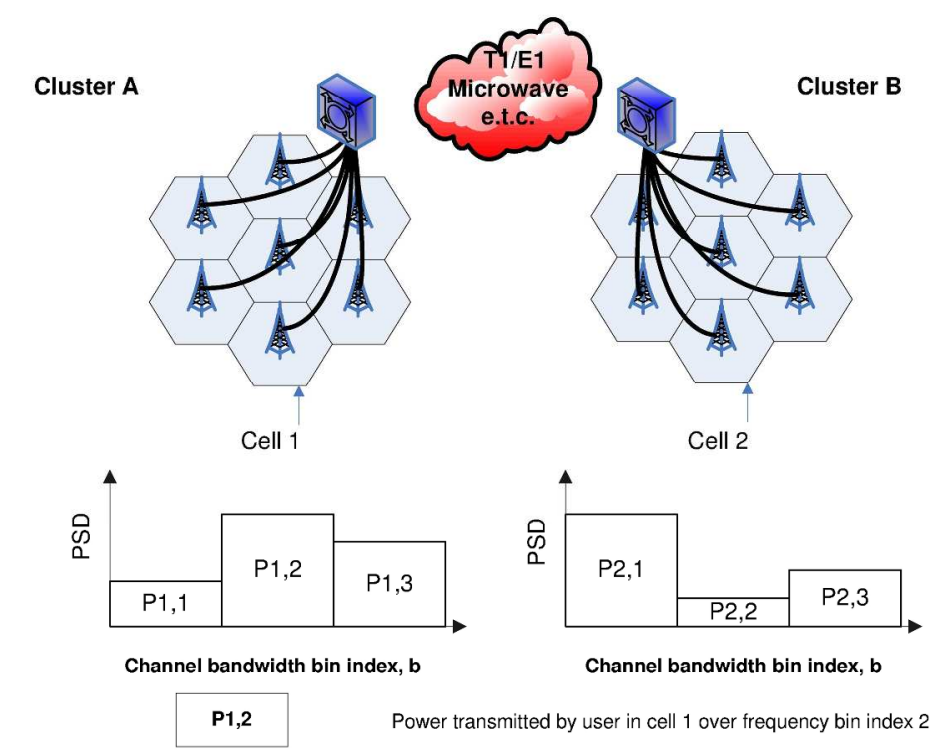

$279 \times 361 \mathrm{~mm}(300 \times 300$ DPI $)$ 


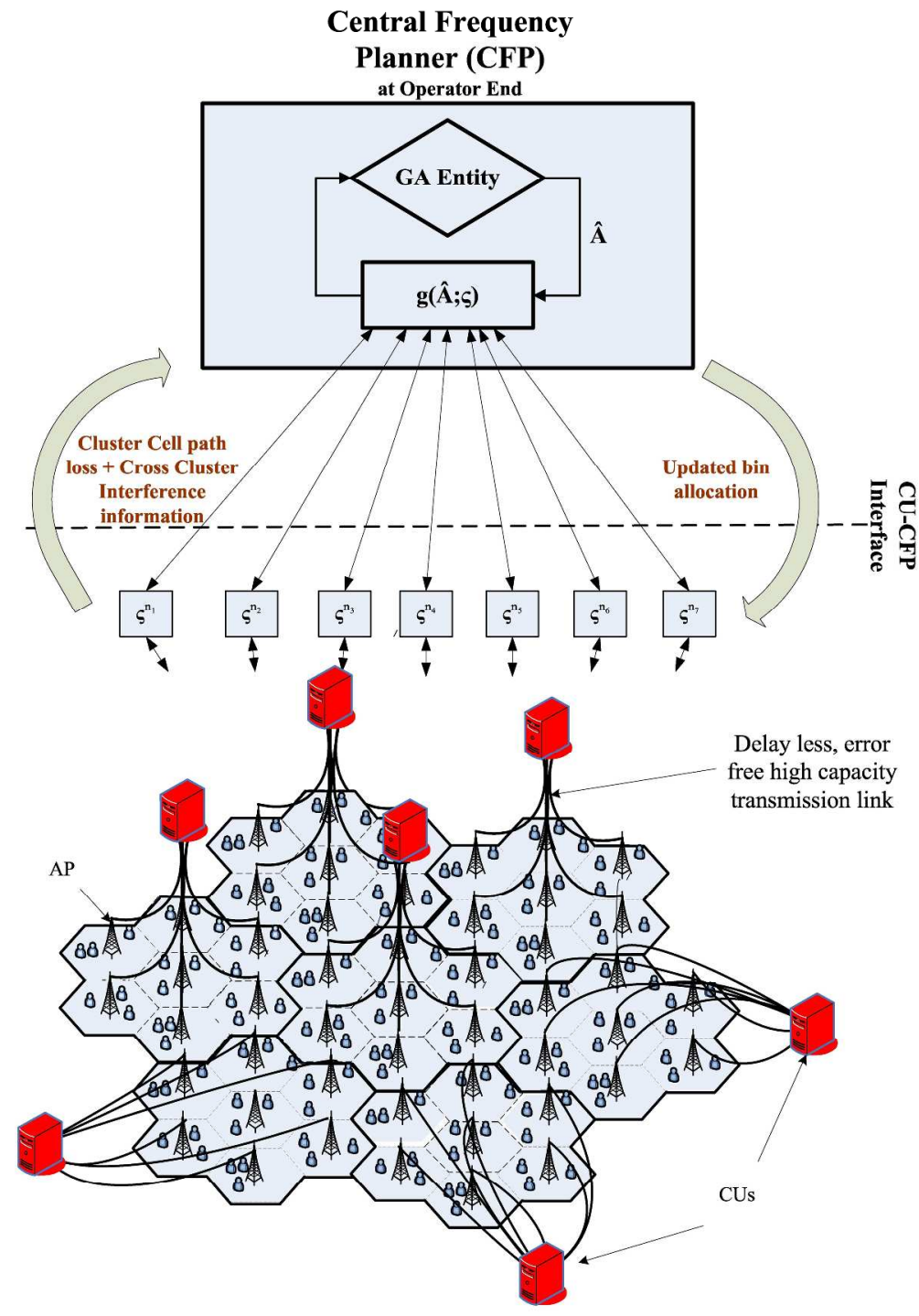

$297 \times 420 \mathrm{~mm}(300 \times 300$ DPI $)$ 


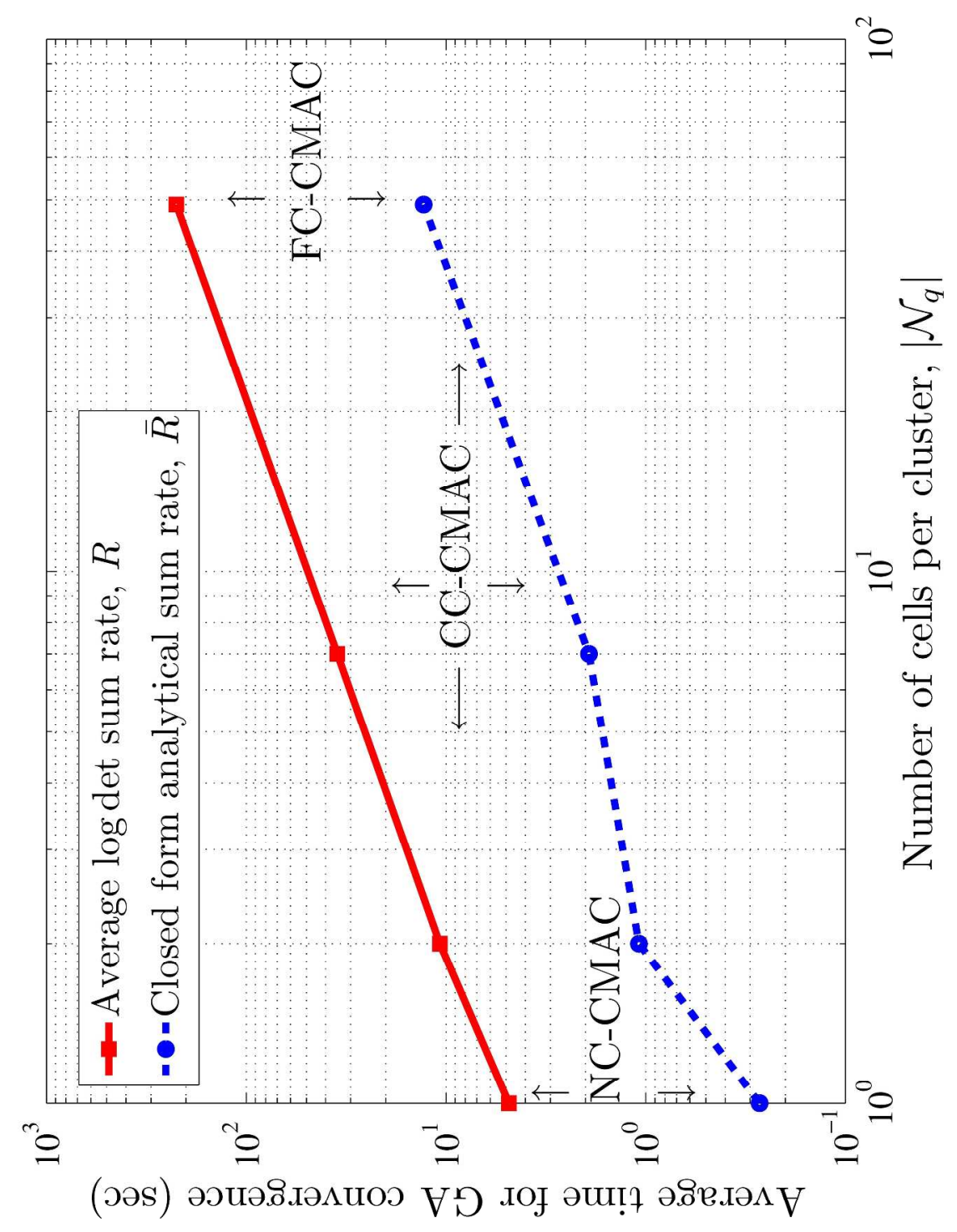

$262 \times 347 \mathrm{~mm}(300 \times 300$ DPI $)$ 


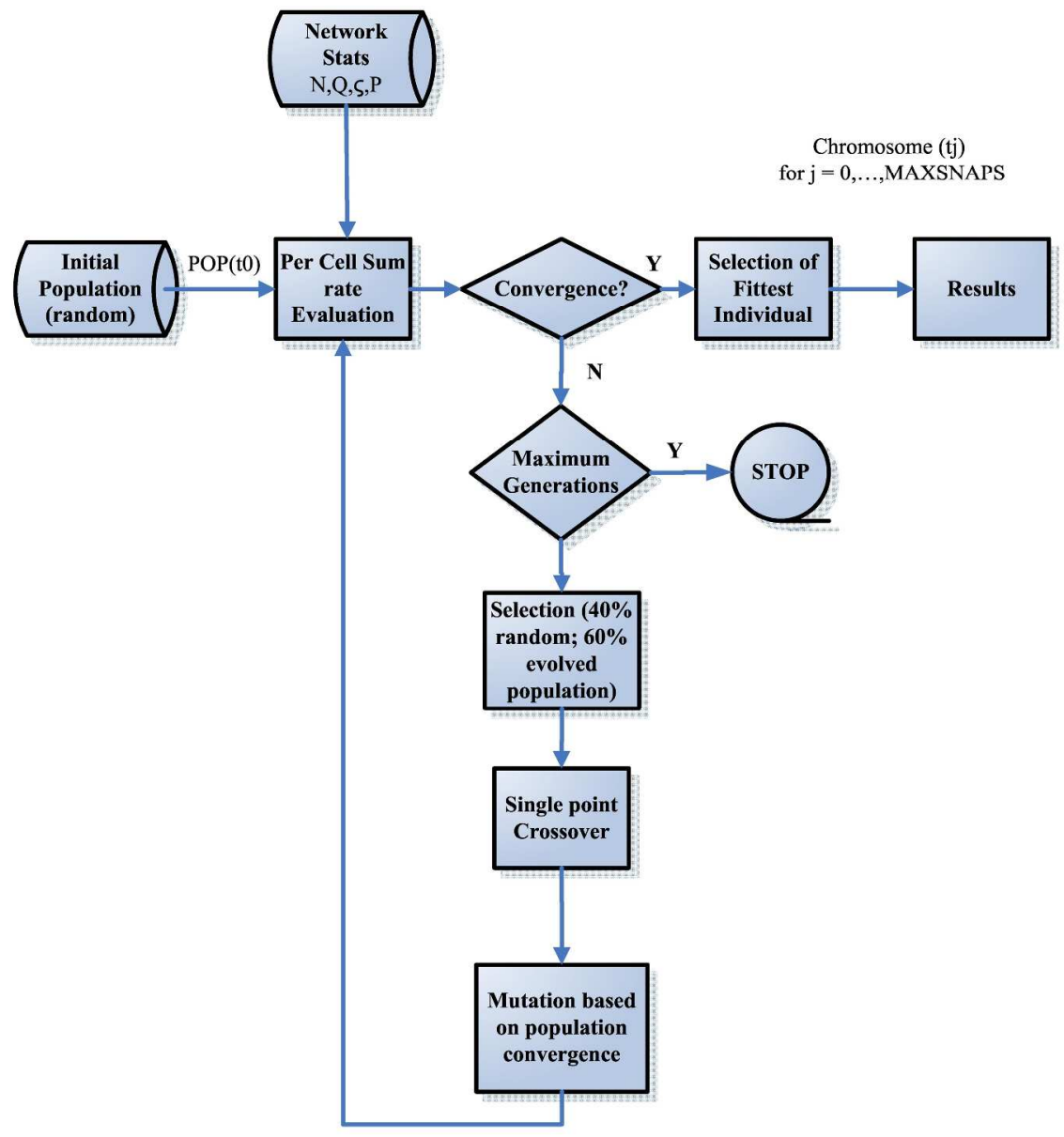

$279 \times 361 \mathrm{~mm}(300 \times 300 \mathrm{DPI})$ 


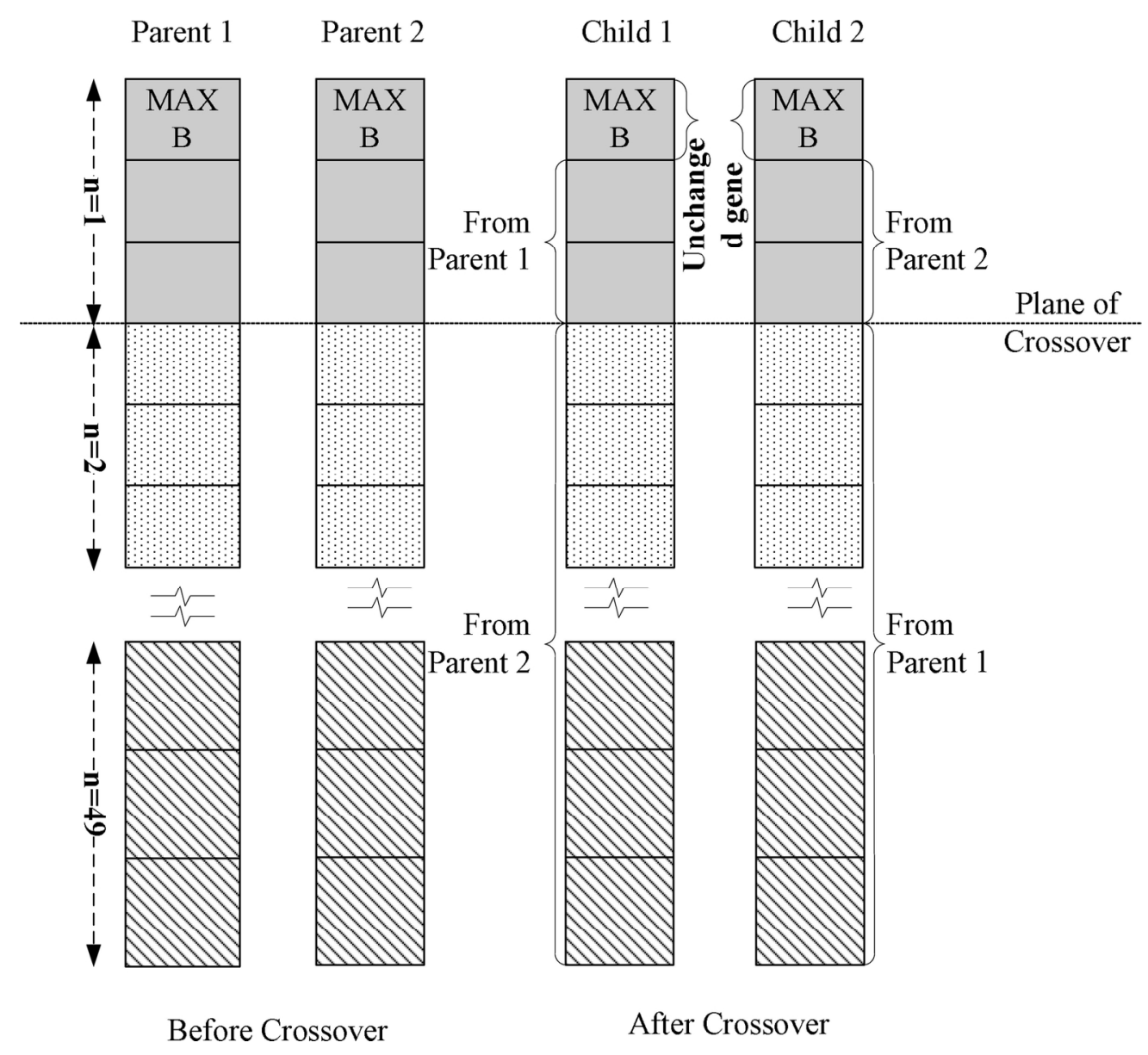

$160 \times 146 \mathrm{~mm}(300 \times 300 \mathrm{DPI})$ 


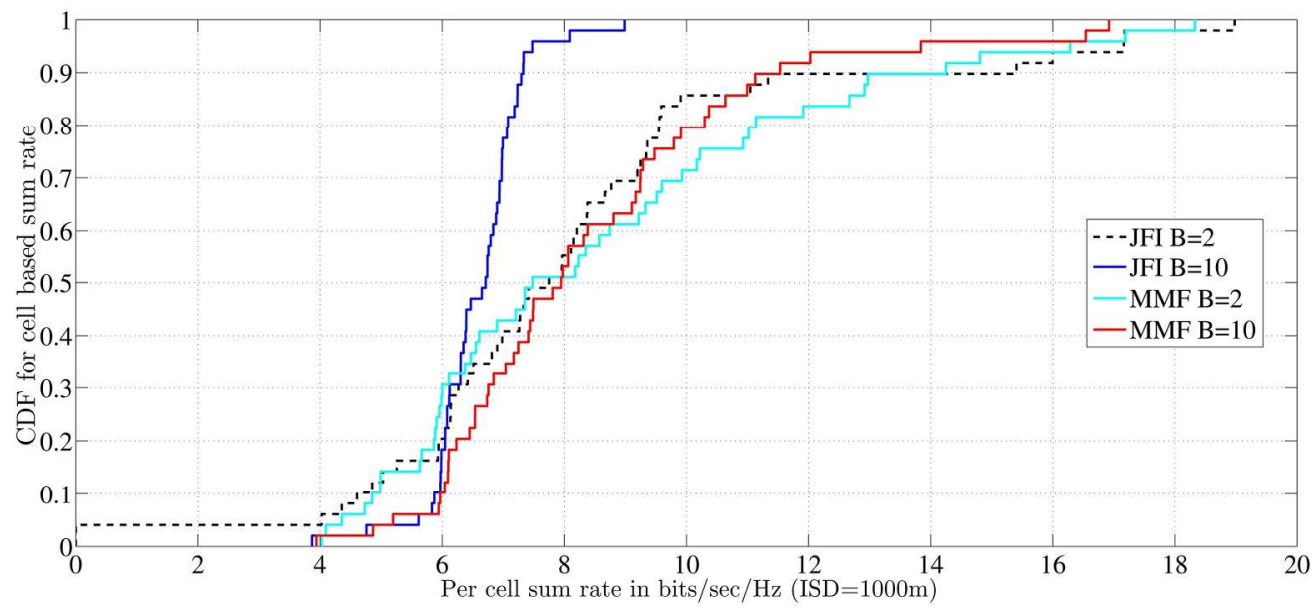

$195 \times 90 \mathrm{~mm}(300 \times 300 \mathrm{DPI})$ 


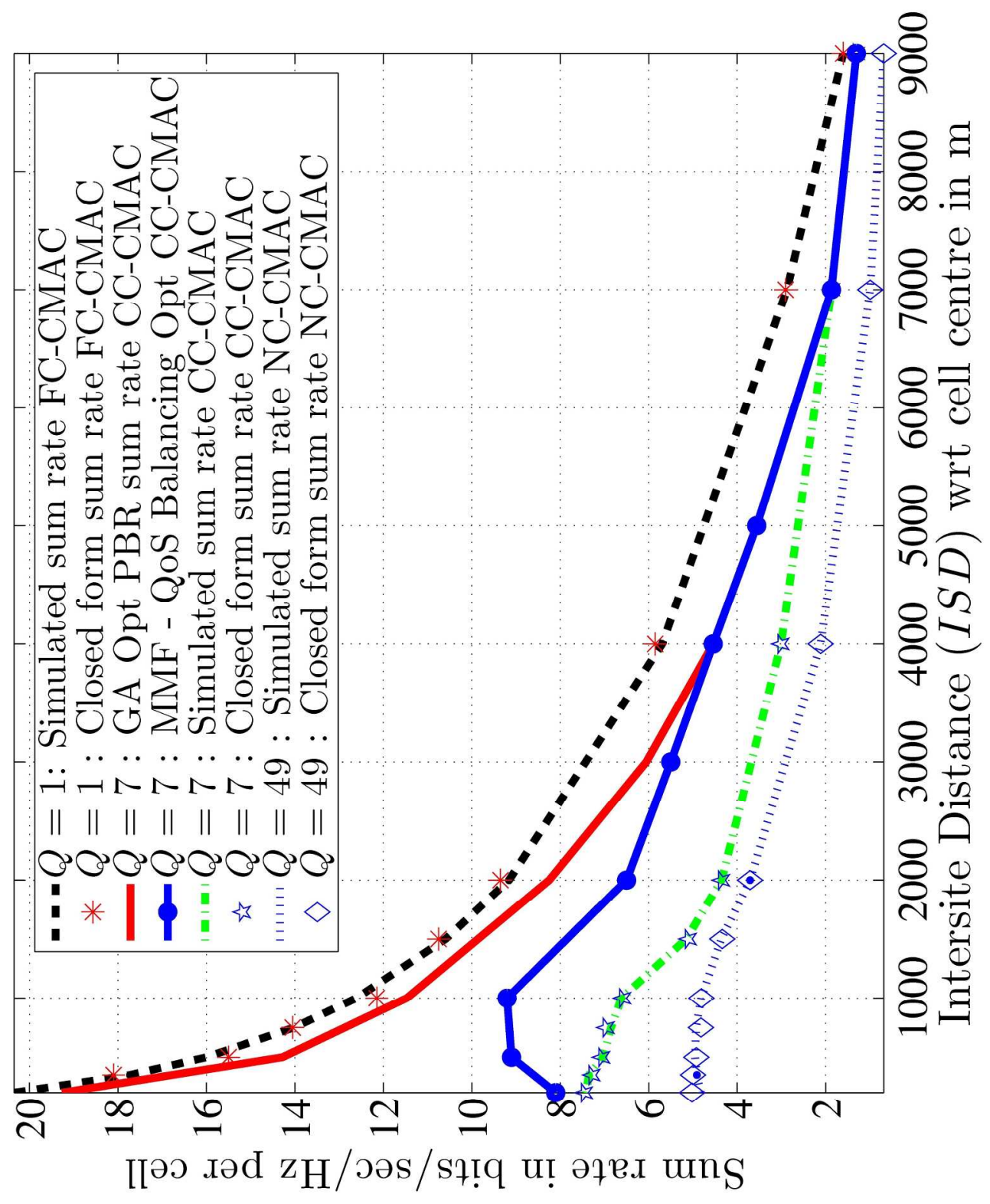

$220 \times 262 \mathrm{~mm}(300 \times 300$ DPI $)$ 


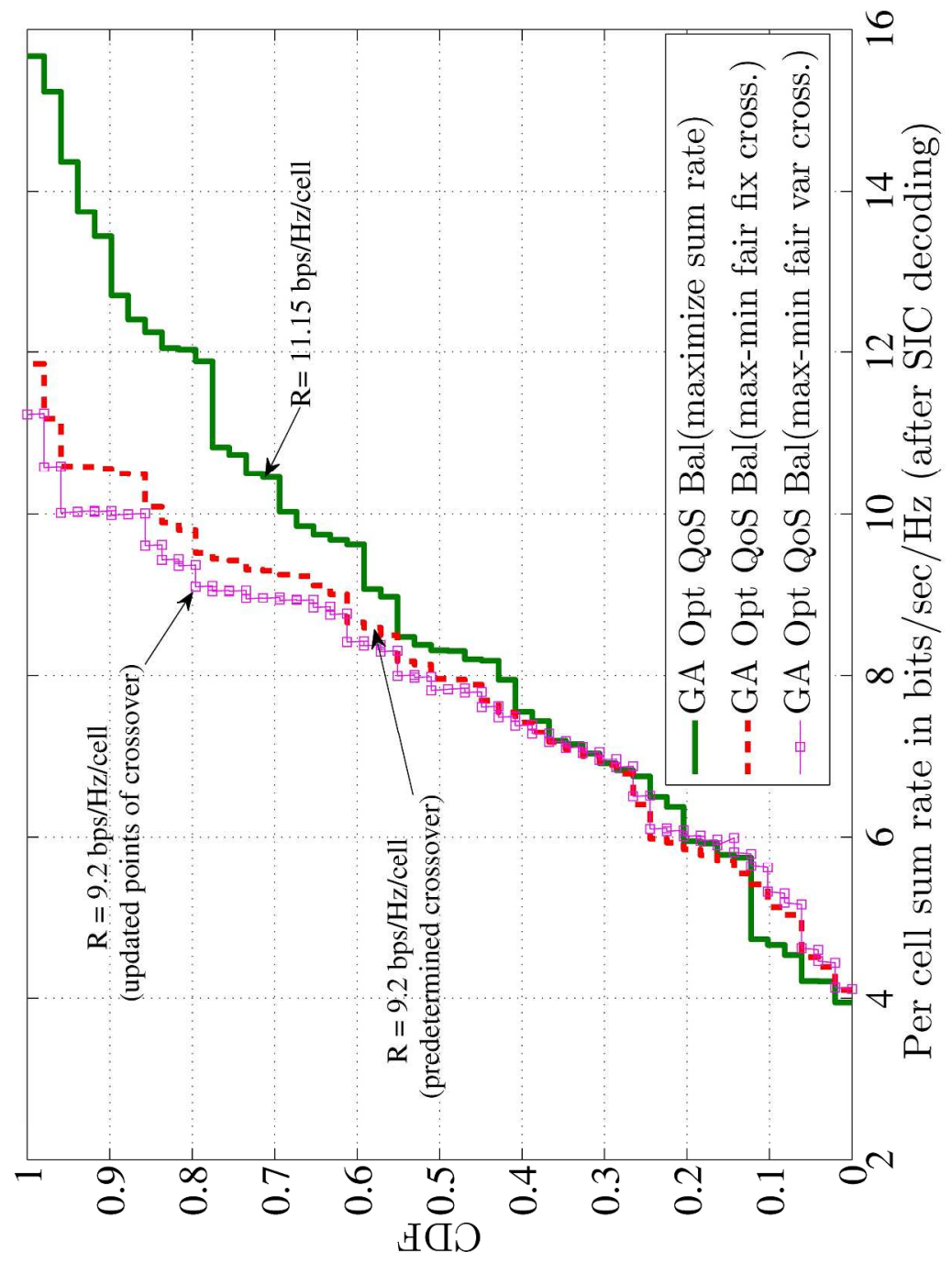

$279 \times 361 \mathrm{~mm}(300 \times 300 \mathrm{DPI})$ 